\title{
Existence and concentration of semi-classical solutions for a nonlinear Maxwell-Dirac system
}

\author{
Yanheng Ding ${ }^{\dagger}, \quad$ Juncheng $\mathrm{Wei}^{\ddagger}$, Tian $\mathrm{Xu}^{\dagger}$ \\ $\dagger$ Institute of Mathematics, AMSS, Chinese Academy of Sciences, \\ 100190 Beijing, China \\ $\ddagger$ Department of Mathematics, The Chinese University of Hong Kong, \\ Shatin, Hong Kong and \\ Department of Mathematics, University of British Columbia \\ Vancouver, B.C., Canada, V6T 1Z2
}

\begin{abstract}
We study the semi-classical ground states of the nonlinear MaxwellDirac system with critical/subcritical nonlinearities:

$$
\left\{\begin{array}{l}
\alpha \cdot(i \hbar \nabla+q(x) \mathbf{A}(x)) w-a \beta w-\omega w-q(x) \phi(x) w=f(x,|w|) w \\
-\Delta \phi=q(x)|w|^{2} \\
-\Delta A_{k}=q(x)\left(\alpha_{k} w\right) \cdot \bar{w} \quad k=1,2,3
\end{array}\right.
$$

for $x \in \mathbb{R}^{3}$, where $\mathbf{A}=\left(A_{1}, A_{2}, A_{3}\right)$ is the magnetic field, $\phi$ is the electron field, $q$ is the changing pointwise charge distribution. We develop a variational argument to establish the existence of least energy solutions for $\hbar$ small. We also describe the concentration phenomena of the solutions as $\hbar \rightarrow 0$.
\end{abstract}

Mathematics Subject Classifications (2000): 35 Q40, 49J35.

Keywords: nonlinear Maxwell-Dirac system, semiclassical states, concentration.

\section{Introduction and main result}

The Maxwell-Dirac system, which have been widely considered in literature (see [1], [15], [17], [21], [23], [24], [29] and references therein), is fundamental in the relativistic description of spin $1 / 2$ particles. It represents the timeevolution of fast (relativistic) electrons and positrons within external and self-consistent generated electromagnetic field. 
The linear Dirac equation coupling to the electromagnetic field whose gauge potentials are $\mathbf{A}=\left(A_{1}, A_{2}, A_{3}\right), \phi$ is

$$
i \hbar \frac{\partial \psi}{\partial t}=\left(\sum_{k=1}^{3} \alpha_{k}\left(-i c \hbar \partial_{k}-q A_{k}\right)\right) \psi+q \phi \psi+m c^{2} \beta \psi,
$$

here $\psi(t, x) \in \mathbb{C}^{4}, c$ denote the speed of light, $q$ denotes the charge of the particle, $m>0$ the mass of the electron, and $\hbar$ denotes Planck's constant. Furthermore, $\alpha_{1}, \alpha_{2}, \alpha_{3}$ and $\beta$ are $4 \times 4$ complex matrices:

$$
\beta=\left(\begin{array}{cc}
I & 0 \\
0 & -I
\end{array}\right), \quad \alpha_{k}=\left(\begin{array}{cc}
0 & \sigma_{k} \\
\sigma_{k} & 0
\end{array}\right), \quad k=1,2,3,
$$

with

$$
\sigma_{1}=\left(\begin{array}{cc}
0 & 1 \\
1 & 0
\end{array}\right), \quad \sigma_{2}=\left(\begin{array}{cc}
0 & -i \\
i & 0
\end{array}\right), \quad \sigma_{3}=\left(\begin{array}{cc}
1 & 0 \\
0 & -1
\end{array}\right),
$$

and $\mathbf{A}: \mathbb{R} \times \mathbb{R}^{3} \rightarrow \mathbb{R}^{3}, \phi: \mathbb{R} \times \mathbb{R}^{3} \rightarrow \mathbb{R}$, and we have used $\alpha=\left(\alpha_{1}, \alpha_{2}, \alpha_{3}\right)$, $\alpha \cdot \nabla=\sum_{k=1}^{3} \alpha_{k} \partial_{k}$, and $\alpha \cdot \mathbf{V}=\sum_{k=1}^{3} \alpha_{k} V_{k}$ for any vector $\mathbf{V} \in \mathbb{C}^{3}$.

If we set

$$
\psi(t, x)=w(t, x) e^{i S(t, x) / \hbar},
$$

where $(w, S): \mathbb{R} \times \mathbb{R}^{3} \rightarrow \mathbb{C}^{4} \times \mathbb{R}$, the Lagrangian density relative to (1.1) is given by (hereafter for $u, v \in \mathbb{C}^{4}, u \bar{v}$ denotes the inner product of $u$ and $v$ )

$$
\begin{aligned}
\mathcal{L}_{0}=\frac{1}{2}[i \hbar & \frac{\partial w}{\partial t} \bar{w}-|w|^{2} \frac{\partial S}{\partial t}-\alpha \cdot(-i c \hbar \nabla+c \nabla S-q \mathbf{A}) w \bar{w} \\
& \left.-q \phi|w|^{2}-m c^{2}(\beta w) \bar{w}\right] .
\end{aligned}
$$

Further, one considers the Lagrangian density of the electromagnetic field $\mathbf{E}, \mathbf{H}$

$$
\mathcal{L}_{1}=\frac{c\left(\mathbf{E}^{2}-\mathbf{H}^{2}\right)}{8 \pi}
$$

and $\mathbf{E}, \mathbf{H}$ are related to $\mathbf{A}, \phi$ by

$$
\mathbf{E}=-\frac{1}{c} \frac{\partial \mathbf{A}}{\partial t}-\nabla \phi, \quad \mathbf{H}=\nabla \times \mathbf{A} .
$$

Then the total action is given by

$$
\mathcal{S}=\iint \mathcal{L}_{0}+\mathcal{L}_{1}
$$

Making the variation of $\mathcal{S}$ with respect to $w, S, \phi$ and $\mathbf{A}$ respectively, we get

$$
i \hbar \partial_{t} w-\partial_{t} S w-\alpha \cdot(-i c \hbar \nabla+c \nabla S-q \mathbf{A}) w-q \phi w-m c^{2} \beta w=0
$$




$$
\begin{gathered}
\partial_{t}|w|^{2}+c \sum_{k=1}^{3} \partial_{k}\left(\left(\alpha_{k} w\right) \bar{w}\right)=0, \\
-\frac{c}{4 \pi} \nabla \cdot\left(\frac{1}{c} \partial_{t} \mathbf{A}+\nabla \phi\right)=q|w|^{2}, \\
q(\alpha w) \bar{w}-\frac{c}{4 \pi} \partial_{t}\left(\frac{1}{c} \partial_{t} \mathbf{A}+\nabla \phi\right)-\frac{c}{4 \pi} \nabla \times(\nabla \times \mathbf{A})=0
\end{gathered}
$$

where $(\alpha w) \bar{w}:=\left(\left(\alpha_{1} w\right) \bar{w},\left(\alpha_{2} w\right) \bar{w},\left(\alpha_{3} w\right) \bar{w}\right)$.

One is interested in finding stationary solutions of (1.4)-(1.7), which have the form

$$
\left\{\begin{array}{l}
\psi(t, x)=w(x) e^{i \theta t / \hbar}, \quad \theta \in \mathbb{R}, \quad w: \mathbb{R}^{3} \rightarrow \mathbb{C}^{4}, \\
\mathbf{A}=\mathbf{A}(x), \quad \phi=\phi(x) \quad \text { in } \mathbb{R}^{3} .
\end{array}\right.
$$

For notation convenience, one shall denote $A_{0}=\phi$. If $\left(\psi, \mathbf{A}, A_{0}\right)$ is a stationary solution of (1.4)-(1.7), then $\left(w, \mathbf{A}, A_{0}\right)$ is a solution of

$$
\left\{\begin{array}{c}
\alpha \cdot(i \hbar \nabla+Q \mathbf{A}) w-a \beta w-\omega w-Q A_{0} w=0, \\
-\Delta A_{k}=4 \pi Q\left(\alpha_{k} w\right) \bar{w}, \quad k=0,1,2,3,
\end{array}\right.
$$

where $a=m c>0, \omega \in \mathbb{R}, Q=q / c$ and $\alpha_{0}:=I$.

The existence of stationary solution of (1.8) has been an open problem for a long time, see [19]. Using variational methods Esteban, Georgiev and Séré [16] proved the existence of regular stationary solutions of the form $\psi(t, x)=w(x) e^{i \omega t}$ with $\omega \in(0, a)$. On the other hand, in [21], Garrett Lisi gave numerical evidence of the existence of bounded states for $\omega \in$ $(-a, a)$. After that, Abenda in [1] obtained the existence result of solitary wave solutions for $\omega \in(-a, a)$.

For small $\hbar$, the solitary waves are referred to as semi-classical states. To describe the translation from quantum to classical mechanics, the existence of solutions $w_{\hbar}, \hbar$ small, possesses an important physical interest. Sparber and Markowich, see [26], studied the existence and asymptotic description of the semiclassical solution of the Cauchy problem for Maxwell-Dirac system as $\hbar \rightarrow 0$, and obtained the asymptotic approximation as $O(\sqrt{\hbar})$.

In this paper we are interested in the existence and concentration phenomenon of stationary semi-classical solutions to the system with

- the changing pointwise charge distribution $Q(x)$ including the constant $q$ as a special one;

- the subcritical self-coupling nonlinearity of the form

$$
\sum_{j=1}^{J} W_{j}(x)|w|^{p_{j}-2} w \quad\left(2<p_{j}<3,1 \leq j \leq J\right) ;
$$


- the critical self-coupling nonlinearity.

More precisely, we consider the system, writing $\varepsilon=\hbar$,

$$
\left\{\begin{array}{l}
\alpha \cdot(i \varepsilon \nabla+Q(x) \mathbf{A}) w-a \beta w-\omega w-Q(x) A_{0} w=f(x,|w|) w \\
-\Delta A_{k}=4 \pi Q(x)\left(\alpha_{k} w\right) \bar{w} \quad k=0,1,2,3 .
\end{array}\right.
$$

Firstly, consider the subcritical case where $f(x, s)=\sum_{j=1}^{J} W_{j}(x) s^{p_{j}-2}$, we assume:

$\left(Q_{0}\right) Q \in C^{0,1}\left(\mathbb{R}^{3}\right)$ with $Q(x) \geq 0$ a.e. on $\mathbb{R}^{3}$;

$\left(P_{0}\right) W_{j} \in C^{0,1}\left(\mathbb{R}^{3}\right)$ with $\inf W_{j}>0$ for $j=1,2, \cdots, J$, and there is $j_{0} \in$ $\{1,2, \cdots, J\}$ such that

$$
\limsup _{|x| \rightarrow \infty} W_{j_{0}}(x)<\max _{x \in \mathbb{R}^{3}} W_{j_{0}}(x),
$$

and there is $x_{0} \in \mathscr{W}_{j_{0}}$ makes $W_{j}\left(x_{0}\right) \geq W_{j}(x)$ for all $|x| \geq R$, some $R$ large and $j \neq j_{0}$, where

$$
\mathscr{W}_{j_{0}}=\left\{x \in \mathbb{R}^{3}: W_{j_{0}}(x)=\max _{x \in \mathbb{R}^{3}} W_{j_{0}}(x)\right\} .
$$

$\left(P_{1}\right) 2<p_{1} \leq p_{2} \leq \cdots \leq p_{J}<3$.

Denoted by $m_{j}=\max _{x \in \mathbb{R}^{3}} W_{j}(x)$,

$$
\begin{aligned}
& \mathscr{W}_{j_{0}}^{1}:=\bigcup_{\substack{j=1 \\
j \neq j_{0}}}^{J}\left\{x \in \mathscr{W}_{j_{0}}: W_{j}(x) \geq W_{j}\left(x_{0}\right)\right\}, \\
& \mathscr{W}_{j_{0}}^{2}:=\bigcup_{\substack{j=1 \\
j \neq j_{0}}}^{J}\left\{x \notin \mathscr{W}_{j_{0}}: W_{j}(x)>W_{j}\left(x_{0}\right)\right\}, \\
& \mathscr{W}_{0}:=\left\{x \in \mathbb{R}^{3}: W_{j}(x)=m_{j}, j=1, \cdots J\right\},
\end{aligned}
$$

and $\mathscr{W}=\mathscr{W}_{j_{0}}^{1} \cup \mathscr{W}_{j_{0}}^{2}$. Under $\left(P_{0}\right)$, we find $\mathscr{W} \not \equiv \emptyset$, and particularly, for the case $\mathscr{W}_{0} \neq \emptyset$ we would have $\mathscr{W}_{j_{0}}^{2}=\emptyset$ and $\mathscr{W}=\mathscr{W}_{j_{0}}^{1}$.

Our results would be

Theorem 1.1. Assume that $\omega \in(-a, a),\left(Q_{0}\right)$ and $\left(P_{0}\right)-\left(P_{1}\right)$ are satisfied. Then for all $\varepsilon>0$ small,

(i) The subcritical system (1.9) has at least one least energy solution $w_{\varepsilon} \in$ $W^{1, q}$ for all $q \geq 2$. In addition, if $Q, W_{j} \in C^{1,1}\left(\mathbb{R}^{3}\right)$ the solutions will be in $C^{1}$ class. 
(ii) The set of all least energy solutions is compact in $W^{1, q}$ for all $q \geq 2$.

(iii) There is a maximum point $x_{\varepsilon}$ of $\left|w_{\varepsilon}\right|$ with $\lim _{\varepsilon \rightarrow 0} \operatorname{dist}\left(x_{\varepsilon}, \mathscr{W}\right)=0$ such that, for some $C, c>o$

$$
\left|w_{\varepsilon}(x)\right| \leq C \exp \left(-\frac{c}{\varepsilon}\left|x-x_{\varepsilon}\right|\right) .
$$

(iv) Setting $u_{\varepsilon}(x):=w_{\varepsilon}\left(\varepsilon x+x_{\varepsilon}\right)$, for any sequence $x_{\varepsilon} \rightarrow \bar{x}$ as $\varepsilon \rightarrow 0$, $u_{\varepsilon}$ converges uniformly to a least energy solution of (the limit equation)

$$
i \alpha \cdot \nabla u-a \beta u-\omega u=\sum_{j=1}^{J} W_{j}(\bar{x})|u|^{p_{j}-2} u .
$$

In particular, if $\mathscr{W}_{0} \neq \emptyset$, then $\lim _{\varepsilon \rightarrow 0} \operatorname{dist}\left(x_{\varepsilon}, \mathscr{W}_{0}\right)=0$ and $u_{\varepsilon}$ converges uniformly to a least energy solution of (the limit equation)

$$
i \alpha \cdot \nabla u-a \beta u-\omega u=\sum_{j=1}^{J} m_{j}|u|^{p_{j}-2} u .
$$

Next we consider the Maxwell-Dirac systems involving the critical exponent of the relevant Sobolev embedding. We would treat the subcritical perturbation of the form $f(x, s)=P(x) g(s)+W(x) s$ for $s \geq 0$. Writing $G(|w|):=\int_{0}^{|w|} g(s) s d s$, we make the following hypotheses:

$\left(g_{1}\right) g(0)=0, g \in C^{1}(0, \infty), g^{\prime}(s)>0$ for $s>0$, and there exist $p \in(2,3)$, $c_{1}>0$ such that $g(s) \leq c_{1}\left(1+s^{p-2}\right)$ for $s \geq 0$;

$\left(g_{2}\right)$ there exist $\sigma>2, \theta>2$ and $c_{0}>0$ such that $c_{0} s^{\sigma} \leq G(s) \leq \frac{1}{\theta} g(s) s^{2}$ for all $s>0$.

A typical example is the power function $g(s)=s^{\sigma-2}$. For describing the charge distribution and external fields, set

$$
\mathcal{R}_{\sigma}:=\left(\frac{S^{3 / 2} c_{0}^{2 /(\sigma-2)}}{6 \gamma}\right)^{(\sigma-2) / 2},
$$

where $S$ denotes the best Sobolev embedding constant: $S|u|_{L^{6}}^{2} \leq|\nabla u|_{L^{2}}^{2}$ for all $u \in H^{1}\left(\mathbb{R}^{3}\right), \sigma$ and $c_{0}$ are the constants form $\left(g_{2}\right)$, and $\gamma$ is the least energy of the ground state for the superlinear subcritical equation (which exists, see [14])

$$
i \alpha \cdot \nabla u-a \beta u-\omega u=|u|^{\sigma-2} u .
$$

Denoting $m:=\max _{x \in \mathbb{R}^{3}} P(x), m_{\infty}=\limsup _{|x| \rightarrow \infty} P(x), l:=\max _{x \in \mathbb{R}^{3}} W(x)$, we will use the following hypotheses: 
$\left(P_{2}\right) P, W \in C^{0,1}\left(\mathbb{R}^{3}\right)$ with $\inf P>0, \inf W>0$ and

$$
m_{\infty}<m, \quad \limsup _{|x| \rightarrow \infty} W(x) \leq l ;
$$

$\left(P_{3}\right) m_{\infty}^{-1} \cdot l^{\sigma-2}<\mathcal{R}_{\sigma}$.

Set additionally,

$$
\mathscr{P}:=\left\{x \in \mathbb{R}^{3}: P(x)=m, W(x)=l\right\} .
$$

Our result reads as

Theorem 1.2. Assume that $\omega \in(-a, a),\left(g_{1}\right)-\left(g_{2}\right),\left(Q_{0}\right)$ and $\left(P_{2}\right)-\left(P_{3}\right)$ are satisfied. Then for all $\varepsilon>0$ small,

(i) The critical system (1.9) has at least one least energy solution $w_{\varepsilon} \in$ $\cap_{q \geq 2} W_{\text {loc }}^{1, q}\left(\mathbb{R}^{3}\right) \cap L^{\infty}\left(\mathbb{R}^{3}\right)$. In addition, if $Q, P, W \in C^{1,1}\left(\mathbb{R}^{3}\right)$ the solutions will be in $C^{1}$ class.

(ii) The set of all least energy solutions is compact in $H^{1}$.

(iii) Assume that $\mathscr{P} \neq \emptyset$. Then there is a maximum point $x_{\varepsilon}$ of $\left|w_{\varepsilon}\right|$ with $\lim _{\varepsilon \rightarrow 0} \operatorname{dist}\left(x_{\varepsilon}, \mathscr{P}\right)=0$ such that $u_{\varepsilon}(x):=w_{\varepsilon}\left(\varepsilon x+x_{\varepsilon}\right)$ converges in $H^{1}$ to a least energy solution of (the limit equation)

$$
i \alpha \cdot \nabla u-a \beta u-\omega u=m g(|u|) u+l|u| u .
$$

(iv) $\left|w_{\varepsilon}(x)\right| \leq C \exp \left(-\frac{c}{\varepsilon}\left|x-x_{\varepsilon}\right|\right)$ for some $C, c>0$.

It is standard that (1.9) is equivalent to, letting $u(x)=w(\varepsilon x)$

$$
\left\{\begin{array}{l}
\alpha \cdot\left(i \nabla+Q_{\varepsilon} \mathbf{A}_{\varepsilon}\right) u-a \beta u-\omega u-Q_{\varepsilon} A_{\varepsilon, 0} u=f(\varepsilon x,|u|) u, \\
-\Delta A_{\varepsilon, k}=\varepsilon^{2} 4 \pi Q_{\varepsilon} J_{k} \quad k=0,1,2,3,
\end{array}\right.
$$

where $Q_{\varepsilon}(x)=Q(\varepsilon x), P_{\varepsilon}(x)=P(\varepsilon x), \mathbf{A}_{\varepsilon}(x)=\mathbf{A}(\varepsilon x), A_{\varepsilon, k}(x)=A_{k}(\varepsilon x)$, $k=0,1,2,3$, and

$$
J_{k}=\left(\alpha_{k} u\right) \bar{u} \text { for } k=0,1,2,3 .
$$

In fact, with the variable substitution: $x \mapsto x / \varepsilon$, we are going to focus on studying the equivalent problem (1.13). Our argument is variational: the semiclassical solutions are obtained as critical points of an energy functional $\Phi_{\varepsilon}$ associated to the equivalent problem (1.13).

There have been a large number of works on existence and concentration phenomenon of semi-classical states of nonlinear Schrödinger-Poisson systems arising in the non-relativistic quantum mechanics, see, for example, $[3,4,7]$ and their references. It is quite natural to ask if certain similar results 
can be obtain for nonlinear Maxwell-Dirac systems arising in the relativistic quantum mechanics. Mathematically, the two systems possess different variational structures, the Mountain-Pass and the Linking structures respectively. The problems in Maxwell-Dirac systems are difficult because they are strongly indefinite in the sense that both the negative and positive parts of the spectrum of Dirac operator are unbounded and consist of essential spectrums. As far as the authors know there have been no results on the existence and concentration phenomenon of semiclassical solutions to nonlinear Maxwell-Dirac systems.

Very recently, one of the authors, jointly with co-authors, developed an argument to obtain some results on existence and concentration of semiclassical solutions for nonlinear Dirac equations but not for Maxwell-Dirac system, see $[11,12,13]$. Compared with the papers, difficulty arises in the Maxwell-Dirac system because of the presence of nonlocal terms $A_{\varepsilon, k}$, $k=0,1,2,3$. In order to overcome this obstacle, we use cut-off arguments. Roughly speaking, an accurate uniformly boundness estimates on $(C)_{c}$-sequences of the associate energy functional $\Phi_{\varepsilon}$ enables us to introduce a new functional $\widetilde{\Phi}_{\varepsilon}$ by virtue of the cut-off technique so that $\widetilde{\Phi}_{\varepsilon}$ has the same least energy solutions as $\Phi_{\varepsilon}$ and can be dealt with more easily, in particular, the influence of these nonlocal terms can be reduced as $\varepsilon \rightarrow 0$. In addition, for obtaining the exponential decay, since the Kato's inequality seems not work well in the present situation, we handle, instead of considering $\Delta|u|$ as in [11], the square of $|u|$, that is $\Delta|u|^{2}$, with the help of identity (4.16), and then describe the decay at infinity in a subtle way.

\section{The variational framework}

\subsection{The functional setting and notations}

In this section we discuss the variational setting for the equivalent system (1.13). Throughout the paper we assume $0 \in \mathscr{P}$ and $0 \in \mathscr{W}$ without loss of generality.

In the sequel, by $|\cdot|_{q}$ we denote the usual $L^{q}$-norm, and $(\cdot, \cdot)_{2}$ the usual $L^{2}$-inner product. Let $H_{0}=i \alpha \cdot \nabla-a \beta-\omega$ denote the self-adjoint operator on $L^{2} \equiv L^{2}\left(\mathbb{R}^{3}, \mathbb{C}^{4}\right)$ with domain $\mathcal{D}\left(H_{0}\right)=H^{1} \equiv H^{1}\left(\mathbb{R}^{3}, \mathbb{C}^{4}\right)$. It is well know that $\sigma\left(H_{0}\right)=\sigma_{c}\left(H_{0}\right)=\mathbb{R} \backslash(-a-\omega, a-\omega)$ where $\sigma(\cdot)$ and $\sigma_{c}(\cdot)$ denote the spectrum and the continuous spectrum. Thus the space $L^{2}$ possesses the orthogonal decomposition:

$$
L^{2}=L^{+} \oplus L^{-}, \quad u=u^{+}+u^{-}
$$

so that $H_{0}$ is positive definite (resp. negative definite) in $L^{+}$(resp. $L^{-}$). Let $E:=\mathcal{D}\left(\left|H_{0}\right|^{1 / 2}\right)=H^{1 / 2}$ (see $\left.[10,14]\right)$ be equipped with the inner product

$$
\langle u, v\rangle=\Re\left(\left|H_{0}\right|^{1 / 2} u,\left|H_{0}\right|^{1 / 2} v\right)_{2}
$$


and the induced norm $\|u\|=\langle u, u\rangle^{1 / 2}$, where $\left|H_{0}\right|$ and $\left|H_{0}\right|^{1 / 2}$ denote respectively the absolute value and the square root of $\left|H_{0}\right|$. Since $\sigma\left(H_{0}\right)=$ $\mathbb{R} \backslash(-a-\omega, a-\omega)$, one has

$$
(a-|\omega|)|u|_{2}^{2} \leq\|u\|^{2} \quad \text { for all } u \in E .
$$

Note that this norm is equivalent to the usual $H^{1 / 2}$-norm, hence $E$ embeds continuously into $L^{q}$ for all $q \in[2,3]$ and compactly into $L_{l o c}^{q}$ for all $q \in[1,3)$. It is clear that $E$ possesses the following decomposition

$$
E=E^{+} \oplus E^{-} \text {with } E^{ \pm}=E \cap L^{ \pm},
$$

orthogonal with respect to both $(\cdot, \cdot)_{2}$ and $\langle\cdot, \cdot\rangle$ inner products. This decomposition induce also a natural decomposition of $L^{p}$, hence there is $d_{p}>0$ such that

$$
d_{p}\left|u^{ \pm}\right|_{p}^{p} \leq|u|_{p}^{p} \text { for all } u \in E .
$$

Let $\mathcal{D}^{1,2} \equiv \mathcal{D}^{1,2}\left(\mathbb{R}^{3}, \mathbb{R}\right)$ be the completion of $C_{c}^{\infty}\left(\mathbb{R}^{3}, \mathbb{R}\right)$ with respect the Dirichlet norm

$$
\|u\|_{\mathcal{D}}^{2}=\int|\nabla u|^{2} d x .
$$

Then (1.13) can be reduced to a single equation with a non-local term. Actually, by $\left(Q_{0}\right)$, for any $u \in E$ one has $Q_{\varepsilon}|u|^{2} \in L^{6 / 5}$ and there holds

$$
\begin{aligned}
\left|\int Q_{\varepsilon}(x) J_{k} \cdot v d x\right| & \leq\left(\left.\left.\int\left|Q_{\varepsilon}(x)\right| u\right|^{2}\right|^{6 / 5} d x\right)^{5 / 6}\left(\int|v|^{6}\right)^{1 / 6} \\
& \leq\left.\left. S_{0}^{-1 / 2}\left|Q_{\varepsilon}\right| u\right|^{2}\right|_{6 / 5}\|v\|_{\mathcal{D}}
\end{aligned}
$$

where $S_{0}$ is the Sobolev embedding constant: $S_{0}|u|_{6}^{2} \leq\|u\|_{\mathcal{D}}^{2}$ for all $u \in \mathcal{D}^{1,2}$. Hence there exists a unique $A_{\varepsilon, u}^{k} \in \mathcal{D}^{1,2}$ for $k=0,1,2,3$ such that

$$
\int \nabla A_{\varepsilon, u}^{k} \nabla v d x=\varepsilon^{2} 4 \pi \int Q_{\varepsilon}(x) J_{k} \cdot v d x
$$

for all $v \in \mathcal{D}^{1,2}$. It follows that $A_{\varepsilon, u}^{k}$ satisfies the Poisson equation

$$
-\Delta A_{\varepsilon, u}^{k}=\varepsilon^{2} 4 \pi Q_{\varepsilon}(x) J_{k}
$$

and there holds

$$
A_{\varepsilon, u}^{k}(x)=\varepsilon^{2} \int \frac{Q_{\varepsilon}(y) J_{k}(y)}{|x-y|} d y=\frac{\varepsilon^{2}}{|x|} *\left(Q_{\varepsilon} J_{k}\right) .
$$

Substituting $A_{\varepsilon, u}^{k}, k=0,1,2,3$ in (1.13), we are led to the equation

$$
H_{0} u-Q_{\varepsilon}(x) A_{\varepsilon, u}^{0} u+\sum_{k=1}^{3} Q_{\varepsilon}(x) \alpha_{k} A_{\varepsilon, u}^{k} u=f(\varepsilon x,|u|) u .
$$


Denote $F(x,|u|)=\int_{0}^{|u|} f(x, s) s d s$ and $F_{\varepsilon}(x,|u|)=F(\varepsilon x,|u|)$. Note that

$$
F_{\varepsilon}(x,|u|)=\left\{\begin{array}{l}
\sum_{j=1}^{J} \frac{1}{p_{j}} W_{j}(\varepsilon x)|u|^{p_{j}} \quad \text { in the subcritical case, } \\
P_{\varepsilon}(x) G(|u|)+\frac{1}{3} W_{\varepsilon}(x)|u|^{3} \quad \text { in the critical case. }
\end{array}\right.
$$

On $E$ we define the functional

$$
\Phi_{\varepsilon}(u)=\frac{1}{2}\left(\left\|u^{+}\right\|^{2}-\left\|u^{-}\right\|^{2}\right)-\Gamma_{\varepsilon}(u)-\Psi_{\varepsilon}(u)
$$

for $u=u^{+}+u^{-}$, where

$$
\begin{gathered}
\Gamma_{\varepsilon}(u)=\frac{1}{4} \int Q_{\varepsilon}(x) A_{\varepsilon, u}^{0}(x) J_{0} d x-\frac{1}{4} \sum_{k=1}^{3} \int Q_{\varepsilon}(x) A_{\varepsilon, u}^{k} J_{k} d x, \\
\Psi_{\varepsilon}(u)=\int F_{\varepsilon}(x,|u|) d x .
\end{gathered}
$$

\subsection{Technical results}

In this subsection, we shall introduce some lemmas that related to the functional $\Phi_{\varepsilon}$.

Lemma 2.1. Under the hypotheses on $f(x, s)$, one has $\Phi_{\varepsilon} \in C^{2}(E, \mathbb{R})$ and any critical point of $\Phi_{\varepsilon}$ is a solution of (1.13).

Proof. Clearly, $\Psi_{\varepsilon} \in C^{2}(E, \mathbb{R})$. It remains to check that $\Gamma_{\varepsilon} \in C^{2}(E, \mathbb{R})$. It suffices to show that, for any $u, v \in E$,

$$
\begin{gathered}
\left|\Gamma_{\varepsilon}(u)\right| \leq \varepsilon^{2} C_{1}|Q|_{\infty}^{2}\|u\|^{4}, \\
\left|\Gamma_{\varepsilon}^{\prime}(u) v\right| \leq \varepsilon^{2} C_{2}|Q|_{\infty}^{2}\|u\|^{3}\|v\|, \\
\left|\Gamma_{\varepsilon}^{\prime \prime}(u)[v, v]\right| \leq \varepsilon^{2} C_{3}|Q|_{\infty}^{2}\|u\|^{2}\|v\|^{2} .
\end{gathered}
$$

Observe that one has, by (2.5) and (2.6) with $v=A_{\varepsilon, u}^{k}$,

$$
\left|A_{\varepsilon, u}^{k}\right|_{6} \leq S_{0}^{-1 / 2}\left\|A_{\varepsilon, u}^{k}\right\|_{\mathcal{D}} \leq \varepsilon^{2} C_{1}|Q|_{\infty}\|u\|^{2} .
$$

This, together with the Hölder inequality (with $r=6, r^{\prime}=6 / 5$ ), implies

(2.9). Note that $\Gamma_{\varepsilon}^{\prime}(u) v=\left.\frac{d}{d t} \Gamma_{\varepsilon}(u+t v)\right|_{t=0}$, so

$$
\begin{aligned}
\Gamma_{\varepsilon}^{\prime}(u) v= & \frac{\varepsilon^{2}}{2} \iint \frac{Q_{\varepsilon}(x) Q_{\varepsilon}(y)}{|x-y|}\left(J_{0}(x) \Re\left[\alpha_{0} u \bar{v}(y)\right]+J_{0}(y) \Re\left[\alpha_{0} u \bar{v}(x)\right]\right. \\
& \left.-\sum_{k=1}^{3}\left(J_{k}(x) \Re\left[\alpha_{k} u \bar{v}(y)\right]+J_{k}(y) \Re\left[\alpha_{k} u \bar{v}(x)\right]\right)\right) d y d x \\
= & \int\left(Q_{\varepsilon} A_{\varepsilon, u}^{0} \Re\left[\alpha_{0} u \bar{v}\right]-\sum_{k=1}^{3} Q_{\varepsilon} A_{\varepsilon, u}^{k} \Re\left[\alpha_{k} u \bar{v}\right]\right)
\end{aligned}
$$


which, together with the Hölder inequality and (2.12), shows (2.10). Similarly,

$$
\begin{aligned}
\Gamma_{\varepsilon}^{\prime \prime}(u)[v, v]=\int Q_{\varepsilon} & \left(A_{\varepsilon, u}^{0} J_{k}^{v}-\sum_{k=1}^{3} A_{\varepsilon, u}^{k} J_{k}^{v}\right) \\
& +2 \varepsilon^{2} \iint \frac{Q_{\varepsilon}(x) Q_{\varepsilon}(y)}{|x-y|}\left[\left(\Re\left[\alpha_{0} u \bar{v}(x)\right]\right)\left(\Re\left[\alpha_{0} u \bar{v}(y)\right]\right)\right. \\
& \left.-\sum_{k=1}^{3}\left(\Re\left[\alpha_{k} u \bar{v}(x)\right]\right)\left(\Re\left[\alpha_{k} u \bar{v}(y)\right]\right)\right]
\end{aligned}
$$

where $J_{k}^{u}=\alpha_{k} u \bar{u}$ and $J_{k}^{v}=\alpha_{k} v \bar{v}$, and one gets (2.11).

Now it is a standard to verify that critical points of $\Phi_{\varepsilon}$ are solutions of (1.13).

We show further the following

Lemma 2.2. For every $\varepsilon>0, \Gamma_{\varepsilon}$ is nonnegative and weakly sequentially lower semi-continuous.

Proof. It is not difficult to see

$$
J_{0}(x) J_{0}(y)-\sum_{k=1}^{3} J_{k}(x) J_{k}(y) \geq 0,
$$

(see an argument of [16]). Hence (see (2.7))

$$
\Gamma_{\varepsilon}(u)=\frac{\varepsilon^{2}}{4} \iint \frac{Q_{\varepsilon}(x) Q_{\varepsilon}(y)\left(J_{0}(x) J_{0}(y)-\sum_{k=1}^{3} J_{k}(x) J_{k}(y)\right)}{|x-y|} d x d y \geq 0 .
$$

And if $u_{n} \rightarrow u$ in $E$, then $u_{n} \rightarrow u$ a.e., and, with (2.14) and Fatou's lemma,

$$
\Gamma_{\varepsilon}(u) \leq \liminf _{n \rightarrow \infty} \Gamma_{\varepsilon}\left(u_{n}\right) .
$$

as required.

In virtue of the assumptions $\left(g_{1}\right)-\left(g_{2}\right)$, for any $\delta>0$, there exist $r_{\delta}>$ $0, c_{\delta}>0$ and $c_{\delta}^{\prime}>0$ such that

$$
\left\{\begin{array}{l}
g(s)<\delta \text { for all } 0 \leq s \leq r_{\delta} \\
G(s) \geq c_{\delta} s^{\theta}-\delta s^{2} \text { for all } s \geq 0 \\
G(s) \leq \delta s^{2}+c_{\delta}^{\prime} s^{p} \text { for all } s \geq 0
\end{array}\right.
$$

and

$$
\widehat{G}(s):=\frac{1}{2} g(s) s^{2}-G(s) \geq \frac{\theta-2}{2 \theta} g(s) s^{2} \geq \frac{\theta-2}{2} G(s) \geq c_{\theta} s^{\sigma}
$$


for all $s \geq 0$, where $c_{\theta}=c_{0}(\theta-2) / 2$.

Set, for $r>0, B_{r}=\{u \in E:\|u\| \leq r\}$, and for $e \in E^{+}$

$$
E_{e}:=E^{-} \oplus \mathbb{R}^{+} e
$$

with $\mathbb{R}^{+}=[0,+\infty)$.

Lemma 2.3. For all $\varepsilon \in(0,1]$, $\Phi_{\varepsilon}$ possess the linking structure:

1) There exist $r>0$ and $\tau>0$, both independent of $\varepsilon$, such that $\left.\Phi_{\varepsilon}\right|_{B_{r}^{+}} \geq 0$ and $\left.\Phi_{\varepsilon}\right|_{S_{r}^{+}} \geq \tau$.

2) For any $e \in E^{+} \backslash\{0\}$, there exist $R=R_{e}>0$ and $C=C_{e}>0$ both independent of $\varepsilon$ such that for all $\varepsilon>0$ there hold $\Phi_{\varepsilon}(u)<0$ for all $u \in E_{e} \backslash B_{R}$ and $\max \Phi_{\varepsilon}\left(E_{e}\right) \leq C$.

Proof. We verify the critical case (the subcritical case can be checked similarly). Recall that $|u|_{p}^{p} \leq C_{p}\|u\|^{p}$ for all $u \in E$ by Sobolev embedding theorem. 1) follows easily because, for $u \in E^{+}$and $\delta>0$ small enough

$$
\begin{aligned}
\Phi_{\varepsilon}(u) & =\frac{1}{2}\|u\|^{2}-\Gamma_{\varepsilon}(u)-\Psi_{\varepsilon}(u) \\
& \geq \frac{1}{2}\|u\|^{2}-\varepsilon^{2} C_{1}|Q|_{\infty}^{2}\|u\|^{4}-|P|_{\infty}\left(\delta|u|_{2}^{2}+c_{\delta}^{\prime}|u|_{p}^{p}\right)-\frac{|W|_{\infty}}{3}|u|_{3}^{3}
\end{aligned}
$$

with $C_{1}, C_{p}$ independent of $u$, and $p>2$.

For checking 2), take $e \in E^{+} \backslash\{0\}$. In virtue of (2.4), for $u=s e+v \in E_{e}$, one gets

$$
\begin{aligned}
\Phi_{\varepsilon}(u) & =\frac{1}{2}\|s e\|^{2}-\frac{1}{2}\|v\|^{2}-\Gamma_{\varepsilon}(u)-\Psi_{\varepsilon}(u) \\
& \leq \frac{1}{2} s^{2}\|e\|^{2}-\frac{1}{2}\|v\|^{2}-\frac{d_{3} \inf W}{3} s^{3}|e|_{3}^{3}
\end{aligned}
$$

proving the conclusion.

Recall that a sequence $\left\{u_{n}\right\} \subset E$ is called to be a $(P S)_{c}$ sequence for functional $\Phi \in C^{1}(E, \mathbb{R})$ if $\Phi\left(u_{n}\right) \rightarrow c$ and $\Phi^{\prime}\left(u_{n}\right) \rightarrow 0$, and is called to be $(C)_{c}$ sequence for $\Phi$ if $\Phi\left(u_{n}\right) \rightarrow c$ and $\left(1+\left\|u_{n}\right\|\right) \Phi^{\prime}\left(u_{n}\right) \rightarrow 0$. It is clear that if $\left\{u_{n}\right\}$ is a $(P S)_{c}$ sequence with $\left\{\left\|u_{n}\right\|\right\}$ bounded then it is also a $(C)_{c}$ sequence. Below we are going to study $(C)_{c}$ sequences for $\Phi_{\varepsilon}$ but firstly we observe the following

Lemma 2.4. Let $\left\{u_{n}\right\} \subset E \backslash\{0\}$ be bounded in $L^{\sigma}$. Then $\left\{\frac{A_{\varepsilon, u_{n}}^{k}}{\varepsilon^{2}\left\|u_{n}\right\|}\right\}$ is bounded in $L^{6}$ uniformly in $\varepsilon \in(0,1]$, for $k=0,1,2,3$. 
Proof. Set $v_{n}=\frac{u_{n}}{\left\|u_{n}\right\|}$. Notice that $A_{\varepsilon, u_{n}}^{k}$ satisfies the equation

$$
-\Delta A_{\varepsilon, u_{n}}^{k}=\varepsilon^{2} 4 \pi Q_{\varepsilon}(x)\left(\alpha_{k} u_{n}\right) \overline{u_{n}},
$$

hence,

$$
-\Delta \frac{A_{\varepsilon, u_{n}}^{k}}{\left\|u_{n}\right\|}=\varepsilon^{2} 4 \pi Q_{\varepsilon}(x)\left(\alpha_{k} u_{n}\right) \overline{v_{n}} .
$$

Observe that $\left\|v_{n}\right\|=1, E$ embeds continuously into $L^{q}$ for $q \in[2,3]$, and

$$
\begin{aligned}
\left|\int Q_{\varepsilon}(x) \alpha_{k} u_{n} \overline{v_{n}} \cdot \psi d x\right| & \leq|Q|_{\infty}\left|u_{n}\right|_{\sigma}\left|v_{n}\right|_{q}|\psi|_{6} \\
& \leq S_{0}^{-1 / 2}|Q|_{\infty}\left|u_{n}\right|_{\sigma}\left|v_{n}\right|_{q}\|\psi\|_{\mathcal{D}}
\end{aligned}
$$

for any $\psi \in \mathcal{D}^{1,2}\left(\mathbb{R}^{3}, \mathbb{C}^{4}\right)$ and $\frac{1}{\sigma}+\frac{1}{q}+\frac{1}{6}=1$. We obtain

$$
\left\|\frac{A_{\varepsilon, u_{n}}^{k}}{\left\|u_{n}\right\|}\right\|_{\mathcal{D}} \leq \varepsilon^{2} \tilde{C}|Q|_{\infty}\left|u_{n}\right|_{\sigma},
$$

as desired.

We now turn to an estimate on boundness of $(C)_{c}$-sequences which is the key ingredient in the sequel. Recall that, by $\left(g_{1}\right)$, there exist $r_{1}>0$ and $a_{1}>0$ such that

$$
g(s) \leq \frac{a-|\omega|}{2|P|_{\infty}} \text { for all } s \leq r_{1},
$$

and, for $s \geq r_{1}, g(s) \leq a_{1} s^{p-2}$, so $g(s)^{\sigma_{0}-1} \leq a_{2} s^{2}$ with

$$
\sigma_{0}:=\frac{p}{p-2}>3
$$

which, jointly with $\left(g_{2}\right)$, yields (see (2.16))

$$
g(s)^{\sigma_{0}} \leq a_{2} g(s) s^{2} \leq a_{3} \widehat{G}(s) \text { for all } s \geq r_{1} .
$$

Lemma 2.5. For any $\lambda>0$, denoting $I=[0, \lambda]$, there is $\Lambda>0$ independent of $\varepsilon$ such that, for all $\varepsilon \in(0,1]$, any $(C)_{c}$-sequence $\left\{u_{n}^{\varepsilon}\right\}$ of $\Phi_{\varepsilon}$ with $c \in I$, there holds (up to a subsequence)

$$
\left\|u_{n}^{\varepsilon}\right\| \leq \Lambda
$$

for all $n \in \mathbb{N}$. 
Proof. Again we only check the critical case because the subcritical case can be dealt with similarly with some obvious modifications.

Let $\left\{u_{n}^{\varepsilon}\right\}$ be a $(C)_{c}$-sequence of $\Phi_{\varepsilon}$ with $c \in I$. Without loss of generality we may assume that $\left\|u_{n}^{\varepsilon}\right\| \geq 1$. The form of $\Phi_{\varepsilon}$ and the representation (2.13) $\left(\Gamma_{\varepsilon}^{\prime}(u) u=4 \Gamma_{\varepsilon}(u)\right)$ implies that

$$
\begin{aligned}
2 \lambda & >c+o(1)=\Phi_{\varepsilon}\left(u_{n}^{\varepsilon}\right)-\frac{1}{2} \Phi_{\varepsilon}^{\prime}\left(u_{n}^{\varepsilon}\right) u_{n}^{\varepsilon} \\
& =\Gamma_{\varepsilon}\left(u_{n}^{\varepsilon}\right)+\int P_{\varepsilon}(x) \widehat{G}\left(\left|u_{n}^{\varepsilon}\right|\right)+\frac{1}{6} \int W_{\varepsilon}(x)\left|u_{n}^{\varepsilon}\right|^{3}
\end{aligned}
$$

and

$$
\begin{aligned}
o(1) & =\Phi_{\varepsilon}^{\prime}\left(u_{n}^{\varphi}\right)\left(u_{n}^{\varepsilon+}-u_{n}^{\varepsilon-}\right) \\
& =\left\|u_{n}^{\varepsilon}\right\|^{2}-\Gamma_{\varepsilon}^{\prime}\left(u_{n}^{\varepsilon}\right)\left(u_{n}^{\varepsilon+}-u_{n}^{\varepsilon-}\right)-\Re \int f\left(\varepsilon x,\left|u_{n}^{\varepsilon}\right|\right) u_{n}^{\varepsilon} \cdot \overline{u_{n}^{\varepsilon+}-u_{n}^{\varepsilon-}} .
\end{aligned}
$$

By Lemma 2.2, (2.16) and (2.20), $\left\{u_{n}^{\varepsilon}\right\}$ is bounded in $L^{\sigma}$ and $L^{3}$ uniformly in $\varepsilon$ with the upper bound, denoted by $C_{1}$, depending on $\lambda, \sigma, \theta$ and inf $P$. It follows from (2.21) that

$$
o(1)+\left\|u_{n}^{\varepsilon}\right\|^{2} \leq \Gamma_{\varepsilon}^{\prime}\left(u_{n}^{\varepsilon}\right)\left(u_{n}^{\varepsilon+}-u_{n}^{\varepsilon-}\right)+\Re \int f\left(\varepsilon x,\left|u_{n}^{\varepsilon}\right|\right) u_{n}^{\varepsilon} \cdot \overline{u_{n}^{\varepsilon+}-u_{n}^{\varepsilon-}} .
$$

This, together with (2.18),(2.2) and the boundedness of $\left\{u_{n}^{\varepsilon}\right\}$ in $L^{3}$, shows

$$
\begin{aligned}
o(1)+\frac{1}{2}\left\|u_{n}^{\varepsilon}\right\|^{2} \leq & \Gamma_{\varepsilon}^{\prime}\left(u_{n}^{\varepsilon}\right)\left(u_{n}^{\varepsilon+}-u_{n}^{\varepsilon-}\right) \\
& +\Re \int_{\left|u_{n}^{\varepsilon}\right| \geq r_{1}} P_{\varepsilon}(x) g\left(\left|u_{n}^{\varepsilon}\right|\right) u_{n}^{\varepsilon} \cdot \overline{u_{n}^{\varepsilon+}-u_{n}^{\varepsilon-}}+C_{2}
\end{aligned}
$$

with $C_{2}$ independent of $\varepsilon$.

Recall that $\left(g_{1}\right)$ and $\left(g_{2}\right)$ imply $2<\sigma \leq p$. Setting $t=\frac{p \sigma}{2 \sigma-p}$, one sees

$$
2<t<p, \quad \frac{1}{\sigma_{0}}+\frac{1}{\sigma}+\frac{1}{t}=1 .
$$

By Hölder inequality, the fact $\Gamma_{\varepsilon}\left(u_{n}^{\varepsilon}\right) \geq 0,(2.19),(2.20)$, the boundedness of $\left\{\left|u_{n}^{\varepsilon}\right|_{\sigma}\right\}$ uniformly in $\varepsilon$, and the embedding of $E$ to $L^{t}$, we have

$$
\begin{aligned}
& \int_{\left|u_{n}^{\varepsilon}\right| \geq r_{1}} P_{\varepsilon}(x) g\left(\left|u_{n}^{\varepsilon}\right|\right)\left|u_{n}^{\varepsilon}\right|\left|u_{n}^{\varepsilon+}-u_{n}^{\varepsilon-}\right| \\
\leq & |P|_{\infty}\left(\int_{\left|u_{n}^{\varepsilon}\right| \geq r_{1}} g\left(\left|u_{n}^{\varepsilon}\right|\right)^{\sigma_{0}}\right)^{1 / \sigma_{0}}\left(\int\left|u_{n}^{\varepsilon}\right|^{\sigma}\right)^{1 / \sigma}\left(\left|u_{n}^{\varepsilon+}-u_{n}^{\varepsilon-}\right|^{t}\right)^{1 / t} \\
\leq & C_{3}\left\|u_{n}^{\varepsilon}\right\|
\end{aligned}
$$

with $C_{3}$ independent of $\varepsilon$. 
Let $q=\frac{6 \sigma}{5 \sigma-6}$. Then $2<q<3$ and $\frac{1}{\sigma}+\frac{1}{q}+\frac{1}{6}=1$. Set

$$
\zeta= \begin{cases}0 & \text { if } q=\sigma \\ \frac{2(\sigma-q)}{q(\sigma-2)} & \text { if } q<\sigma \\ \frac{3(q-\sigma)}{q(3-\sigma)} & \text { if } q>\sigma\end{cases}
$$

and note that

$$
|u|_{q} \leq\left\{\begin{array}{l}
|u|_{2}^{\zeta} \cdot|u|_{\sigma}^{1-\zeta} \quad \text { if } 2<q \leq \sigma \\
|u|_{3}^{\zeta} \cdot|u|_{\sigma}^{1-\zeta} \quad \text { if } \sigma<q<3 .
\end{array}\right.
$$

By virtue of the Hölder inequality, Lemma 2.2, the boundedness of $\left\{\left|u_{n}^{\varepsilon}\right|_{\sigma}\right\}$ and $\left\{\left|u_{n}^{\varepsilon}\right|_{3}\right\}$, and the embedding of $E$ to $L^{2}$ and $L^{3}$, we obtain that

$$
\begin{aligned}
& \left|\Re \int Q_{\varepsilon}(x) A_{\varepsilon, u_{n}^{\varepsilon}}^{k}\left(\alpha_{k} u_{n}^{\varepsilon}\right) \cdot \overline{u_{n}^{\varepsilon+}-u_{n}^{\varepsilon-}}\right| \\
= & \left|\varepsilon^{2}\left\|u_{n}^{\varepsilon}\right\| \Re \int Q_{\varepsilon}(x) \frac{A_{\varepsilon, u_{n}^{\varepsilon}}^{k}}{\varepsilon^{2}\left\|u_{n}^{\varepsilon}\right\|}\left(\alpha_{k} u_{n}^{\varepsilon}\right) \cdot \overline{u_{n}^{\varepsilon+}-u_{n}^{\varepsilon-}}\right| \\
\leq & \varepsilon^{2}|Q|_{\infty}\left\|u_{n}^{\varepsilon}\right\|\left|\frac{A_{\varepsilon, u_{n}^{\varepsilon}}^{k}}{\varepsilon^{2}\left\|u_{n}^{\varepsilon}\right\|}\right|_{6}\left|u_{n}^{\varepsilon}\right|_{\sigma}\left|u_{n}^{\varepsilon+}-u_{n}^{\varepsilon-}\right|_{q} \\
\leq & \varepsilon^{2} C_{3}\left\|u_{n}^{\varepsilon}\right\|\left|u_{n}^{\varepsilon}\right|_{q} \leq \varepsilon^{2} C_{4}\left\|u_{n}^{\varepsilon}\right\|^{1+\zeta}
\end{aligned}
$$

with $C_{4}$ independent of $\varepsilon$. This, together with the representation of (2.13), implies that

$$
\left|\Gamma_{\varepsilon}^{\prime}\left(u_{n}^{\varepsilon}\right)\left(u_{n}^{\varepsilon+}-u_{n}^{\varepsilon-}\right)\right| \leq C_{5}\left\|u_{n}^{\varepsilon}\right\|^{1+\zeta}
$$

with $C_{5}$ independent of $\varepsilon$.

Now the combination of (2.22), (2.23) and (2.24) shows that

$$
\left\|u_{n}^{\varepsilon}\right\|^{2} \leq M_{0}+M_{1}\left\|u_{n}^{\varepsilon}\right\|+M_{2}\left\|u_{n}^{\varepsilon}\right\|^{1+\zeta}
$$

with $M_{0}, M_{1}$ and $M_{2}$ being independent of $\varepsilon \leq 1$. Therefore, there is $\Lambda \geq 1$ independent of $\varepsilon$ such that

$$
\left\|u_{n}^{\varepsilon}\right\| \leq \Lambda
$$

as desired.

Finally, for the later aim we define the operator $\mathcal{A}_{\varepsilon, k}: E \rightarrow \mathcal{D}^{1,2}\left(\mathbb{R}^{3}\right)$ by $\mathcal{A}_{\varepsilon, k}(u)=A_{\varepsilon, u}^{k}$. We have

Lemma 2.6. For $k=0,1,2,3$,

(1) $\mathcal{A}_{\varepsilon, k}$ maps bounded sets into bounded sets; 
(2) $\mathcal{A}_{\varepsilon, k}$ is continuous.

Proof. Clearly, (1) is a straight consequence of (2.12). (2) follows easily because, for $u, v \in E, \quad A_{\varepsilon, u}^{j}-A_{\varepsilon, v}^{j}$ satisfies

$$
-\Delta\left(A_{\varepsilon, u}^{j}-A_{\varepsilon, v}^{j}\right)=\varepsilon^{2} 4 \pi Q_{\varepsilon}(x)\left[\alpha_{j} u \bar{u}-\alpha_{j} v \bar{v}\right],
$$

consequently,

$$
\begin{aligned}
\left\|A_{\varepsilon, u}^{j}-A_{\varepsilon, v}^{j}\right\|_{\mathcal{D}^{1,2}} & \leq \varepsilon^{2} C|Q|_{\infty}\left|\left(\alpha_{j} u\right) \bar{u}-\left(\alpha_{j} v\right) \bar{v}\right|_{6 / 5} \\
& \leq \varepsilon^{2} C|Q|_{\infty}\left(|u-v|_{12 / 5}|u|_{12 / 5}+|u-v|_{12 / 5}|v|_{12 / 5}\right) \\
& \leq \varepsilon^{2} C_{1}|Q|_{\infty}(\|u-v\| \cdot\|u\|+\|u-v\| \cdot\|v\|) .
\end{aligned}
$$

This implies the desired conclusion.

\section{Preliminary results}

Observe that the non-local term $\Gamma_{\varepsilon}$ is rather complex. The main purpose of this section is, by cut-off arguments, to introduce an auxiliary functional which will simplify our arguments.

In order to prove our main result, we will make use of some results on the following autonomous equations for $u \in H^{1}\left(\mathbb{R}^{3}, \mathbb{C}^{4}\right)$ :

$$
\begin{gathered}
i \alpha \cdot \nabla u-a \beta u-\omega u=\sum_{j=1}^{J} \nu_{j}|u|^{p_{j}-2} u, \\
i \alpha \cdot \nabla u-a \beta u-\omega u=\mu g(|u|) u, \\
i \alpha \cdot \nabla u-a \beta u-\omega u=\mu g(|u|) u+\chi|u| u,
\end{gathered}
$$

where $\vec{\nu}:=\left(\nu_{1}, \ldots, \nu_{J}\right) \in \mathbb{R}^{J}$ with $\nu_{j}>0, j=1, \ldots, J$, and $\mu, \chi>0$.

\subsection{The equation (3.1)}

Its solutions are critical points of the functional

$$
\begin{aligned}
\mathscr{T}_{\vec{\nu}}(u) & :=\frac{1}{2}\left(\left\|u^{+}\right\|^{2}-\left\|u^{-}\right\|^{2}\right)-\sum_{j=1}^{J} \frac{\nu_{j}}{p_{j}} \int_{\mathbb{R}^{3}}|u|^{p_{j}} \\
& =\frac{1}{2}\left(\left\|u^{+}\right\|^{2}-\left\|u^{-}\right\|^{2}\right)-\mathscr{G}_{\vec{\nu}}(u)
\end{aligned}
$$


defined for $u=u^{+}+u^{-} \in E=E^{+} \oplus E^{-}$. Denote the critical set, the least energy, and the set of least energy solutions of $\mathscr{T}_{\vec{\nu}}$ as follows

$$
\begin{aligned}
& \mathscr{K}_{\vec{\nu}}:=\left\{u \in E: \mathscr{T}_{\vec{\nu}}^{\prime}(u)=0\right\}, \\
& \gamma_{\vec{\nu}}:=\inf \left\{\mathscr{T}_{\vec{\nu}}(u): u \in \mathscr{K}_{\vec{\nu}} \backslash\{0\}\right\}, \\
& \mathscr{R}_{\vec{\nu}}:=\left\{u \in \mathscr{K}_{\vec{\nu}}: \mathscr{T}_{\vec{\nu}}(u)=\gamma_{\vec{\nu}},|u(0)|=|u|_{\infty}\right\} .
\end{aligned}
$$

The following lemma is from [14].

Lemma 3.1. There hold the following

i) $\mathscr{K}_{\vec{\nu}} \neq \emptyset, \gamma_{\vec{\nu}}>0$, and $\mathscr{K}_{\vec{\nu}} \subset \bigcap_{q \geq 2} W^{1, q}$;

ii) $\gamma_{\vec{\nu}}$ is attained, and $\mathscr{R}_{\vec{\nu}}$ is compact in $H^{1}\left(\mathbb{R}^{3}, \mathbb{C}^{4}\right)$;

iii) there exist $C, c>0$ such that

$$
|u(x)| \leq C \exp (-c|x|) \text { for all } x \in \mathbb{R}^{3}, u \in \mathscr{R}_{\vec{\nu}} .
$$

Motivated by Ackermann [2] (also see [11, 12, 14]), for a fixed $u \in E^{+}$, let $\varphi_{u}: E^{-} \rightarrow \mathbb{R}$ be defined by

$$
\varphi_{u}(v)=\mathscr{T}_{\vec{\nu}}(u+v) .
$$

Observe that, for any $v, w \in E^{-}$,

$$
\varphi_{u}^{\prime \prime}(v)[w, w]=-\|w\|^{2}-\mathscr{G}_{\vec{\nu}}^{\prime \prime}(u+v)[w, w] \leq-\|w\|^{2} .
$$

In addition

$$
\varphi_{u}(v) \leq \frac{1}{2}\|u\|^{2}-\frac{1}{2}\|v\|^{2} .
$$

Therefore, there exists a unique $\mathscr{J}_{\vec{\nu}}: E^{+} \rightarrow E^{-}$such that

$$
\mathscr{T}_{\vec{\nu}}\left(u+\mathscr{J}_{\vec{\nu}}(u)\right)=\max _{v \in E^{-}} \mathscr{T}_{\vec{\nu}}(u+v) .
$$

Now one introduces the following notations (see, [2]):

$$
\begin{aligned}
& J_{\vec{\nu}}: E^{+} \rightarrow \mathbb{R}, J_{\vec{\nu}}(u)=\mathscr{T}_{\vec{\nu}}\left(u+\mathscr{J}_{\vec{\nu}}(u)\right) ; \\
& \mathscr{M}_{\vec{\nu}}:=\left\{u \in E^{+} \backslash\{0\}: J_{\vec{\nu}}^{\prime}(u) u=0\right\} .
\end{aligned}
$$

We call $J_{\vec{\nu}}$ is the reduction functional for $\mathscr{T}_{\vec{\nu}}$ on $E^{+}$. Plainly, critical points of $J_{\vec{\nu}}$ and $\mathscr{T}_{\vec{\nu}}$ are in one to one correspondence via the injective map $u \rightarrow$ $u+\mathscr{J}_{\vec{\nu}}(u)$ from $E^{+}$into $E$.

It is not difficult to check that, for each $u \in E^{+} \backslash\{0\}$, there is a unique $t=t(u)>0$ such that $t u \in \mathscr{M}_{\vec{\nu}}$ (see $[2,14]$ ). Moreover, letting $u \in \mathscr{M}_{\vec{\nu}}$ be such that $J_{\vec{\nu}}(u)=\gamma_{\vec{\nu}}$, and set $E_{u}=E^{-} \oplus \mathbb{R}^{+} u$, one verifies easily that

$$
\max _{w \in E_{u}} \mathscr{T}_{\vec{\nu}}(w)=J_{\vec{\nu}}(u) .
$$


Clearly, $J_{\vec{\nu}}$ has the Mountain-pass structure, which, together with the above discussion, implies

$$
\gamma_{\vec{\nu}}=\inf \left\{J_{\vec{\nu}}(u): u \in \mathscr{M}_{\vec{\nu}}\right\}=\inf _{\gamma \in \Omega_{\vec{\nu}}} \max _{t \in[0,1]} J_{\vec{\nu}}(\gamma(t)),
$$

where $\Omega_{\vec{\nu}}:=\left\{\gamma \in C\left([0,1], E^{+}\right): \gamma(0)=0, J_{\vec{\nu}}(\gamma(1))<0\right\}$.

In the sequel, for vectors $\vec{a}=\left(a_{1}, \ldots, a_{J}\right), \vec{b}=\left(b_{1}, \ldots, b_{J}\right) \in \mathbb{R}^{J}$, we write $\vec{a} \geq \vec{b}$ if $a_{j} \geq b_{j}$ for $j=1, \ldots, J$, and $\vec{a}>\vec{b}$ if $\vec{a} \geq \vec{b}$ and $a_{j_{0}}>b_{j_{0}}$ for some $j_{0}$. The following lemma is clear.

Lemma 3.2. Let $\vec{\nu}_{k}>0, k=1,2$. If $\vec{\nu}_{1}-\vec{\nu}_{2}>0$ then $\gamma_{\vec{\nu}_{1}}<\gamma_{\vec{\nu}_{2}}$.

\subsection{The equation (3.2)}

Its solutions are critical points of the functional

$$
\begin{aligned}
\mathscr{T}_{\mu}(u) & :=\frac{1}{2}\left(\left\|u^{+}\right\|^{2}-\left\|u^{-}\right\|^{2}\right)-\mu \int G(|u|) \\
& =\frac{1}{2}\left(\left\|u^{+}\right\|^{2}-\left\|u^{-}\right\|^{2}\right)-\mathscr{G}_{\mu}(u)
\end{aligned}
$$

defined for $u=u^{+}+u^{-} \in E=E^{+} \oplus E^{-}$. Denote the critical set, the least energy and the set of least energy solutions of $\mathscr{T}_{\mu}$ as follows

$$
\begin{aligned}
& \mathscr{K}_{\mu}:=\left\{u \in E: \mathscr{T}_{\mu}^{\prime}(u)=0\right\} \\
& \gamma_{\mu}:=\inf \left\{\mathscr{T}_{\mu}(u): u \in \mathscr{K}_{\mu} \backslash\{0\}\right\}, \\
& \mathscr{R}_{\mu}:=\left\{u \in \mathscr{K}_{\mu}: \mathscr{T}_{\mu}(u)=\gamma_{\mu},|u(0)|=|u|_{\infty}\right\} .
\end{aligned}
$$

The following lemma is from [14]

Lemma 3.3. There hold the following:

i) $\mathscr{K}_{\mu} \neq \emptyset, \gamma_{\mu}>0$ and $\mathscr{K}_{\mu} \subset \cap_{q \geq 2} W^{1, q}$;

ii) $\gamma_{\mu}$ is attained and $\mathscr{R}_{\mu}$ is compact in $H^{1}\left(\mathbb{R}^{3}, \mathbb{C}^{4}\right)$;

iii) there exist $C, c>0$ such that $|u(x)| \leq C \exp (-c|x|)$ for all $x \in \mathbb{R}^{3}$ and $u \in \mathscr{R}_{\mu}$.

Now as before we introduce:

$$
\begin{aligned}
& \mathscr{J}_{\mu}: E^{+} \rightarrow E^{-}, \mathscr{T}_{\mu}\left(u+\mathscr{J}_{\mu}(u)\right)=\max _{v \in E^{-}} \mathscr{T}_{\mu}(u+v), \\
& J_{\mu}: E^{+} \rightarrow \mathbb{R}, J_{\mu}(u)=\mathscr{T}_{\mu}\left(u+\mathscr{J}_{\mu}(u)\right), \\
& \mathscr{M}_{\mu}:=\left\{u \in E^{+} \backslash\{0\}: J_{\mu}^{\prime}(u) u=0\right\} .
\end{aligned}
$$


For any $u \in E^{+}$and $v \in E^{-}$, setting $z=v-\mathscr{J}_{\mu}(u)$ and $l(t)=\mathscr{T}_{\mu}(u+$ $\left.\mathscr{J}_{\mu}(u)+t z\right)$, one has $l(1)=\mathscr{T}_{\mu}(u+v), l(0)=\mathscr{T}_{\mu}\left(u+\mathscr{J}_{\mu}(u)\right)$ and $l^{\prime}(0)=0$. Thus $l(1)-l(0)=\int_{0}^{1}(1-t) l^{\prime \prime}(t) d t$. This implies that

$$
\begin{aligned}
& \mathscr{T}_{\mu}(u+v)-\mathscr{T}_{\mu}\left(u+\mathscr{J}_{\mu}(u)\right) \\
= & \int_{0}^{1}(1-t) \mathscr{T}_{\mu}^{\prime \prime}\left(u+\mathscr{J}_{\mu}(u)+t z\right)[z, z] d t \\
= & -\int_{0}^{1}(1-t)\|z\|^{2} d t-\int_{0}^{1}(1-t) \mathscr{G}_{\mu}^{\prime \prime}\left(u+\mathscr{J}_{\mu}(u)+t z\right)[z, z] d t,
\end{aligned}
$$

hence

$$
\begin{aligned}
& \int_{0}^{1}(1-t) \mathscr{G}_{\mu}^{\prime \prime}\left(u+\mathscr{J}_{\mu}(u)+t z\right)[z, z] d t+\frac{1}{2}\|z\|^{2} \\
& =\mathscr{T}_{\mu}\left(u+\mathscr{J}_{\mu}(u)\right)-\mathscr{T}_{\mu}(u+v) .
\end{aligned}
$$

It is not difficult to see that, for each $u \in E^{+} \backslash\{0\}$ there is a unique $t=$ $t(u)>0$ such that $t u \in \mathscr{M}_{\mu}$ and

$$
\gamma_{\mu}=\inf \left\{J_{\mu}(u): u \in \mathscr{M}_{\mu}\right\}=\inf _{e \in E^{+} \backslash\{0\}} \max _{u \in E_{e}} \mathscr{T}_{\mu}(u)
$$

(see [14], [11]).

For the later use, define, for $\sigma \in(2,3)$,

$$
T_{\sigma}:=\inf _{u \in E^{+} \backslash\{0\}} \max _{v \in E^{-}} \frac{\|u\|^{2}-\|v\|^{2}}{|u+v|_{\sigma}^{2}},
$$

and consider the equation

$$
i \alpha \cdot \nabla z-a \beta z-\omega z=|z|^{\sigma-2} z
$$

with the energy functional defined by

$$
S_{\sigma}(z):=\frac{1}{2}\left(\left\|z^{+}\right\|^{2}-\left\|z^{-}\right\|^{2}\right)-\frac{1}{\sigma}|z|_{\sigma}^{\sigma}
$$

and the least energy denoted by $\gamma$. The following lemma is due to [13]: Lemma 4.5.

Lemma 3.4. $T_{\sigma}$ is achieved at some $z$ which is a least energy solution of the equation (3.5). Moreover,

$$
T_{\sigma}=\left(\frac{2 \sigma \gamma}{\sigma-2}\right)^{(\sigma-2) / \sigma} .
$$


Proof. For reader's convenience, we give the sketch of the argument in [13]. Set, for any $u \in E^{+}$and $v \in E^{-}$,

$$
\pi_{u}(v)=\frac{\|u\|^{2}-\|v\|^{2}}{|u+v|_{\sigma}^{2}} \quad \text { and } \quad T_{\sigma}(u)=\max _{v \in E^{-}} \pi_{u}(v) .
$$

If $w \in E^{-}$with $\pi_{u}(w)=T_{\sigma}(u)$, then after tedious computations we have, for $v \in E^{-}$,

$$
\pi_{u}^{\prime}(w) v=0 \quad \text { and } \quad \pi_{u}^{\prime \prime}(w)[v, v]<0 .
$$

Hence, $\pi_{u}$ attains its maximum at a unique point.

Observe that

$$
T_{\sigma}=\inf _{u \in E^{+} \backslash\{0\}} \max _{w \in E_{u}} \frac{\left\|w^{+}\right\|^{2}-\left\|w^{-}\right\|^{2}}{|w|_{\sigma}^{2}} .
$$

If the function

$$
m_{u}(w)=\frac{\left\|w^{+}\right\|^{2}-\left\|w^{-}\right\|^{2}}{|w|_{\sigma}^{2}}
$$

attains its maximum on $E_{u}$ at $w$, setting $\widehat{w}=m_{u}(w)^{1 /(\sigma-2)} w /|w|_{\sigma}$, then $m_{u}(\widehat{w})=m_{u}(w)$ and, for any $v \in E_{u}, m_{u}^{\prime}(\widehat{w}) v=0$.

Consequently,

$$
\gamma \leq S_{\sigma}(\widehat{w})=\frac{\sigma-2}{2 \sigma}\left(m_{u}(\widehat{w})\right)^{\sigma /(\sigma-2)}=\frac{\sigma-2}{2 \sigma}\left(m_{u}(w)\right)^{\sigma /(\sigma-2)},
$$

hence, $T_{\sigma} \geq\left(\frac{2 \sigma \gamma}{\sigma-2}\right)^{(\sigma-2) / \sigma}$.

On the other hand, let $z$ be a least energy solution of (3.5) with $z=$ $z^{+}+z^{-}$and take $u=z^{+}$. One has $m_{u}(z)=|z|_{\sigma}^{\sigma-2}$. Plainly, for $v \in E^{-}$,

$$
\pi_{u}^{\prime}\left(z^{-}\right) v=0 \quad \text { and } \quad \pi_{u}^{\prime \prime}\left(z^{-}\right)[v, v]<0 .
$$

Therefore,

$$
T_{\sigma}\left(z^{+}\right)=\frac{\left\|z^{+}\right\|^{2}-\left\|z^{-}\right\|^{2}}{|z|_{\sigma}^{2}}=|z|_{\sigma}^{\sigma-2}=\left(\frac{2 \sigma \gamma}{\sigma-2}\right)^{(\sigma-2) / \sigma},
$$

completing the proof.

Lemma 3.5. If $g(s)=c_{0} s^{\sigma-2}$, then the corresponding least energy of (3.2) denoted by $\gamma_{\mu}(\sigma)$ satisfies

$$
\gamma_{\mu}(\sigma) \leq\left(\mu c_{0}\right)^{-2 /(\sigma-2)} \gamma
$$


Proof. Now assume $g(s)=c_{0} s^{\sigma-2}$, and denote the corresponding energy functional of (3.2) by

$$
S^{\mu}(u)=\frac{1}{2}\left(\left\|u^{+}\right\|^{2}-\left\|u^{-}\right\|^{2}\right)-\frac{\mu c_{0}}{\sigma} \int|u|^{\sigma} .
$$

Let $z$ be a least energy solution of (3.5) and $u=z^{+}$. Set $e_{q} \in E_{u}$ with $S^{\mu}\left(e_{q}\right)=\max _{w \in E_{u}} S^{\mu}(w)$. Then by Lemma 3.4 ,

$$
\begin{aligned}
\gamma_{\mu}(\sigma) & \leq S^{\mu}\left(e_{q}\right) \\
& =\frac{\sigma-2}{2 \sigma}\left(\mu c_{0}\right)^{-2 /(\sigma-2)}\left(\frac{\left\|e_{q}^{+}\right\|^{2}-\left\|e_{q}^{-}\right\|^{2}}{\left|e_{q}\right|_{\sigma}^{2}}\right)^{\sigma /(\sigma-2)} \\
& \leq \frac{\sigma-2}{2 \sigma}\left(\mu c_{0}\right)^{-2 /(\sigma-2)} T_{\sigma}^{\sigma /(\sigma-2)} \\
& =\left(\mu c_{0}\right)^{-2 /(\sigma-2)} \gamma .
\end{aligned}
$$

as desired.

\subsection{The equation (3.3)}

Its solutions are critical points of the functional

$$
\begin{aligned}
\mathscr{T}_{\mu \chi}^{*}(u) & :=\frac{1}{2}\left(\left\|u^{+}\right\|^{2}-\left\|u^{-}\right\|^{2}\right)-\mu \int G(|u|)-\frac{\chi}{3} \int|u|^{3} \\
& =\frac{1}{2}\left(\left\|u^{+}\right\|^{2}-\left\|u^{-}\right\|^{2}\right)-\mathscr{G}_{\mu \chi}^{*}(u) .
\end{aligned}
$$

defined for $u=u^{+}+u^{-} \in E=E^{+} \oplus E^{-}$. Denote the critical set, the least energy and the set of least energy solutions of $\mathscr{T}_{\mu \chi}^{*}$ as follows

$$
\begin{aligned}
& \mathscr{K}_{\mu \chi}^{*}:=\left\{u \in E: \mathscr{T}_{\mu \chi}^{* \prime}(u)=0\right\}, \\
& \gamma_{\mu \chi}^{*}:=\inf \left\{\mathscr{T}_{\mu \chi}^{*}(u): u \in \mathscr{K}_{\mu \chi}^{*} \backslash\{0\}\right\}, \\
& \mathscr{R}_{\mu \chi}^{*}:=\left\{u \in \mathscr{K}_{\mu \chi}^{*}: \mathscr{T}_{\mu \chi}^{*}(u)=\gamma_{\mu \chi}^{*},|u(0)|=|u|_{\infty}\right\} .
\end{aligned}
$$

Firstly we have the following

Lemma 3.6. $\gamma_{\mu \chi}^{*}$ is attained if

$$
\gamma_{\mu \chi}^{*}<\ell^{*}:=\frac{S^{3 / 2}}{6 \chi^{2}}
$$

Proof. Let $\left\{u_{n}\right\}$ be a $(C)_{c}$-sequence with $c=\gamma_{\mu \chi}^{*}$. By the statements in Lemma 2.5, $\left\{u_{n}\right\}$ is bounded in E. By Lion's concentration principle [20], $\left\{u_{n}\right\}$ is either vanishing or non-vanishing. 
Assume that $\left\{u_{n}\right\}$ is vanishing. Then $\left|u_{n}\right|_{s} \rightarrow 0$ for $s \in(2,3)$. By $\left(g_{1}\right)$, $\left(g_{2}\right)$ one gets

$$
c+o(1)=\mathscr{T}_{\mu \chi}^{*}\left(u_{n}\right)-\frac{1}{3} \mathscr{T}_{\mu \chi}^{* \prime}\left(u_{n}\right) u_{n} \geq \frac{1}{6}\left(\left\|u_{n}^{+}\right\|^{2}-\left\|u_{n}^{-}\right\|^{2}\right)
$$

or

$$
\left\|u_{n}^{+}\right\|^{2}-\left\|u_{n}^{-}\right\|^{2} \leq 6 c+o(1)
$$

Similarly,

$$
\int\left|u_{n}\right|^{3} \leq \frac{6 c}{\chi}+o(1)
$$

Moreover,

$$
\left\|u_{n}\right\|^{2}-\chi \cdot \Re \int\left|u_{n}\right| u_{n} \overline{\left(u_{n}^{+}-u_{n}^{-}\right)} \leq o(1) .
$$

Thus,

$$
\left\|u_{n}\right\|^{2} \leq \chi\left|u_{n}\right|_{3}^{2}\left|u_{n}^{+}-u_{n}^{-}\right|_{3}+o(1) .
$$

Observe that $S^{1 / 2}|u|_{3}^{2} \leq\|u\|^{2}$ (see [9]), we have

$$
c \geq \frac{S^{3 / 2}}{6 \chi^{2}}
$$

a contradiction.

Therefore, $\left\{u_{n}\right\}$ is non-vanishing, that is, there exist $r, \delta>0$ and $x_{n} \in \mathbb{R}^{3}$ such that, setting $v_{n}(x)=u_{n}\left(x+x_{n}\right)$, along a subsequence,

$$
\int_{B_{r}(0)}\left|v_{n}\right|^{2} \geq \delta
$$

Without loss of generality we assume $v_{n} \rightarrow v$. Then $v \neq 0$ and is a solution of (3.3). And so $\gamma_{\mu \chi}^{*}$ is attained.

Lemma 3.7. $\gamma_{\mu \chi}^{*}$ is attained if

$$
\mu^{-1} \chi^{\sigma-2}<\mathcal{R}_{\sigma}
$$

Proof. Observe that, for the nonlinearities, we have

$$
\mathscr{G}_{\mu \chi}^{*}(u) \geq \mathscr{G}_{\mu}(u) \geq \frac{\mu c_{0}}{\sigma} \int|u|^{\sigma} .
$$

So, by the reduction process and the min-max scheme, we deduce

$$
\gamma_{\mu \chi}^{*} \leq \gamma_{\mu} \leq \gamma_{\mu}(\sigma)
$$

If

$$
\left(\mu c_{0}\right)^{-2 /(\sigma-2)} \gamma<\ell^{*}
$$

that is, (3.7) is satisfied, then $\gamma_{\mu \chi}^{*}<\ell^{*}$. So $\gamma_{\mu \chi}^{*}$ is attained by Lemma 3.6. 
As a consequence of Lemma 3.7 we have

Lemma 3.8. If (3.7) is satisfied, then

$$
\begin{aligned}
& \mathscr{K}_{\mu \chi}^{*}:=\left\{u \in E: \mathscr{T}_{\mu \chi}^{* \prime}(u)=0\right\} \neq \emptyset, \\
& \gamma_{\mu \chi}^{*}=\inf \left\{\mathscr{T}_{\mu \chi}^{*}(u): u \in \mathscr{K}_{\mu \chi}^{*} \backslash\{0\}\right\}>0,
\end{aligned}
$$

and $\mathscr{R}_{\mu \chi}^{*}:=\left\{u \in \mathscr{K}_{\mu \chi}^{*}: \mathscr{T}_{\mu \chi}^{*}(u)=\gamma_{\mu \chi}^{*},|u(0)|=|u|_{\infty}\right\}$ is compact in $E$.

Set as before the induced map $\mathscr{J}_{\mu \chi}^{*}: E^{+} \rightarrow E^{-}$, the functional $J_{\mu \chi}^{*} \in$ $C^{1}\left(E^{+}, \mathbb{R}\right): J_{\mu \chi}^{*}(u):=\mathscr{T}_{\mu \chi}^{*}\left(u+\mathscr{J}_{\mu \chi}^{*}(u)\right)$ and the manifold $\mathscr{M}_{\mu \chi}^{*}$. The following lemma will be useful to study our problem.

Lemma 3.9. There hold:

1). Let $u \in \mathscr{M}_{\mu \chi}^{*}$ be such that $J_{\mu \chi}^{*}(u)=\gamma_{\mu \chi}^{*}$ and set $E_{u}=E^{-} \oplus \mathbb{R}^{+} u$.

Then

$$
\max _{w \in E_{u}} \mathscr{T}_{\mu \chi}^{*}(w)=J_{\mu \chi}^{*}(u)
$$

2). If $\mu_{1} \leq \mu_{2}$ and $\chi_{1} \leq \chi_{2}$, then

$$
\gamma_{\mu_{1} \chi_{1}}^{*} \geq \gamma_{\mu_{2} \chi_{2}}^{*} .
$$

In addition, if $\max \left\{\mu_{2}-\mu_{1}, \chi_{2}-\chi_{1}\right\}>0$, then

$$
\gamma_{\mu_{1} \chi_{1}}^{*}>\gamma_{\mu_{2} \chi_{2}}^{*} \text {. }
$$

Proof. Since 1) is obvious (see [11, 12]), we only prove 2 ).

Let $u \in \mathscr{L}_{\mu_{1} \chi_{1}}^{*}$ with $\mathscr{T}_{\mu_{1} \chi_{1}}^{*}(u)=\gamma_{\mu_{1} \chi_{1}}^{*}$ and set $e=u^{+}$. Then

$$
\gamma_{\mu_{1} \chi_{1}}^{*}=\mathscr{T}_{\mu_{1} \chi_{1}}^{*}(u)=\max _{w \in E_{e}} \mathscr{T}_{\mu_{1} \chi_{1}}^{*}(w) .
$$

Let $u_{1} \in E_{e}$ be such that $\mathscr{T}_{\mu_{2} \chi_{2}}^{*}\left(u_{1}\right)=\max _{w \in E_{e}} \mathscr{T}_{\mu_{2} \chi_{2}}^{*}(w)$. One has

$$
\begin{aligned}
\gamma_{\mu_{1} \chi_{1}}^{*} & =\mathscr{T}_{\mu_{1} \chi_{1}}^{*}(u) \geq \mathscr{T}_{\mu_{1} \chi_{1}}^{*}\left(u_{1}\right) \\
& =\mathscr{T}_{\mu_{2} \chi_{2}}^{*}\left(u_{1}\right)+\left(\mu_{2}-\mu_{1}\right) \int G\left(\left|u_{1}\right|\right)+\frac{\left(\chi_{2}-\chi_{1}\right)}{3} \int\left|u_{1}\right|^{3} \\
& \geq \gamma_{\mu_{2} \chi_{2}}^{*}+\left(\mu_{2}-\mu_{1}\right) \int G\left(\left|u_{1}\right|\right)+\frac{\left(\chi_{2}-\chi_{1}\right)}{3} \int\left|u_{1}\right|^{3}
\end{aligned}
$$

as desired.

Remark 3.10. Similarly to (3.4) we have, for any $u \in E^{+}$and $v \in E^{-}$,

$$
\begin{aligned}
& \int_{0}^{1}(1-t) \mathscr{G}_{\mu \chi}^{*^{\prime \prime}}\left(u+\mathscr{J}_{\mu \chi}^{*}(u)+t z\right)[z, z] d t+\frac{1}{2}\|z\|^{2} \\
& =\mathscr{T}_{\mu \chi}^{*}\left(u+\mathscr{J}_{\mu \chi}^{*}(u)\right)-\mathscr{T}_{\mu \chi}^{*}(u+v)
\end{aligned}
$$

where $z:=v-\mathscr{J}_{\mu \chi}^{*}(u)$. 


\subsection{Auxiliary functionals}

In order to make the reduction method work for $\Phi_{\varepsilon}$ as $\varepsilon$ small, we circumvent by cutting off the nonlocal terms. We find our current framework is more delicate, since the solutions we look for are at the least energy level and $\Gamma_{\varepsilon}$ is not convex (even for $u$ with $\|u\|$ large). By cutting off the nonlocal terms, and using the reduction method, we are able to find a critical point via an appropriate min-max scheme. The critical point will eventually be shown to be a least energy solution of the original equation when $\varepsilon$ is sufficiently small.

By virtue of $\left(P_{0}\right)$ and $\left(P_{2}\right)$, set $b_{j}=\inf W_{j}$ for $j=1,2, \cdots, J, b=\inf P$ and $d=\inf W$, denote $\vec{b}=\left(b_{1}, \ldots, b_{J}\right)$,

$$
\gamma_{b}=\left\{\begin{array}{l}
\gamma_{\vec{b}} \text { for the subcritical case } \\
\gamma_{b d}^{*} \text { for the critical case }
\end{array}\right.
$$

and let

$$
e_{0} \in\left\{\begin{array}{l}
\mathscr{R}_{\vec{b}} \text { for the subcritical case; } \\
\mathscr{R}_{b d}^{*} \text { for the critical case. }
\end{array}\right.
$$

By the linking structure and the representation of $\Phi_{\varepsilon}$ we have directly

Lemma 3.11. For all $\varepsilon>0, \max _{v \in E_{e_{0}}} \Phi_{\varepsilon}(v) \leq \gamma_{\mathfrak{b}}$.

To introduce the modified functional, by virtue of Lemma 2.5 , for $\lambda=\gamma_{\mathfrak{b}}$ and $I=\left[0, \gamma_{b}\right]$, let $\Lambda \geq 1$ be the associated constant (independent of $\varepsilon$ ). Denote $T:=(\Lambda+1)^{2}$ and let $\eta:[0, \infty) \rightarrow[0,1]$ be a smooth function with $\eta(t)=1$ if $0 \leq t \leq T, \eta(t)=0$ if $t \geq T+1, \max \left|\eta^{\prime}(t)\right| \leq c_{1}$ and $\max \left|\eta^{\prime \prime}(t)\right| \leq c_{2}$. Define

$$
\begin{aligned}
\widetilde{\Phi}_{\varepsilon}(u) & =\frac{1}{2}\left(\left\|u^{+}\right\|^{2}-\left\|u^{-}\right\|^{2}\right)-\eta\left(\|u\|^{2}\right) \Gamma_{\varepsilon}(u)-\Psi_{\varepsilon}(u) \\
& =\frac{1}{2}\left(\left\|u^{+}\right\|^{2}-\left\|u^{-}\right\|^{2}\right)-\mathscr{F}_{\varepsilon}(u)-\Psi_{\varepsilon}(u) .
\end{aligned}
$$

By definition, $\left.\Phi_{\varepsilon}\right|_{B_{T}}=\left.\widetilde{\Phi}_{\varepsilon}\right|_{B_{T}}$. It is easy to see that $0 \leq \mathscr{F}_{\varepsilon}(u) \leq \Gamma_{\varepsilon}(u)$ and

$$
\left|\mathscr{F}_{\varepsilon}^{\prime}(u) v\right| \leq\left|2 \eta^{\prime}\left(\|u\|^{2}\right) \Gamma_{\varepsilon}(u)\langle u, v\rangle\right|+\left|\Gamma_{\varepsilon}^{\prime}(u) v\right|
$$

for $u, v \in E$.

Lemma 3.12. There exists $\varepsilon_{1}>0$ such that, for any $\varepsilon \leq \varepsilon_{1}$, if $\left\{u_{n}^{\varepsilon}\right\}$ is $a(C)_{c}$ sequence of $\widetilde{\Phi}_{\varepsilon}$ with $c \in I$ then $\left\|u_{n}^{\varepsilon}\right\| \leq \Lambda+\frac{1}{2}$, and consequently $\widetilde{\Phi}_{\varepsilon}\left(u_{n}^{\varepsilon}\right)=\Phi_{\varepsilon}\left(u_{n}^{\varepsilon}\right)$. 
Proof. We discuss only the critical case because the subcritical case is easier and can be handled similarly. We repeat the arguments of Lemma 2.5. Let

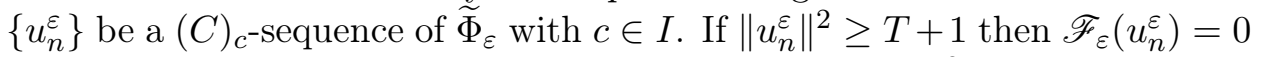
so, as proved in Lemma 2.5, one changes (2.25) by $\left\|u_{n}^{\varepsilon}\right\|^{2} \leq M_{0}+M_{1}\left\|u_{n}^{\varepsilon}\right\|$ and gets $\left\|u_{n}^{\varepsilon}\right\| \leq \Lambda$, a contradiction. Thus we assume that $\left\|u_{n}^{\varepsilon}\right\|^{2} \leq T+1$. Then, using (2.9), $\left|\eta^{\prime}\left(\left\|u_{n}^{\varepsilon}\right\|^{2}\right)\left\|u_{n}^{\varepsilon}\right\|^{2} \Gamma_{\varepsilon}\left(u_{n}^{\varepsilon}\right)\right| \leq \varepsilon^{2} d_{1}$ (here and in the following, by $d_{j}$ we denote positive constants independent of $\varepsilon$ ). Similar to (2.20),

$$
\begin{aligned}
2 \gamma_{\mathrm{b}}>c+o(1) \geq & \left(\eta\left(\left\|u_{n}^{\varepsilon}\right\|^{2}\right)+2 \eta^{\prime}\left(\left\|u_{n}^{\varepsilon}\right\|^{2}\right)\left\|u_{n}^{\varepsilon}\right\|^{2}\right) \Gamma_{\varepsilon}\left(u_{n}^{\varepsilon}\right) \\
& +\int P_{\varepsilon}(x) \widehat{G}\left(\left|u_{n}^{\varepsilon}\right|\right)+\frac{1}{6} \int W_{\varepsilon}(x)\left|u_{n}^{\varepsilon}\right|^{3}
\end{aligned}
$$

which yields

$$
2 \gamma_{\mathrm{b}}+\varepsilon^{2} d_{1}>\eta\left(\left\|u_{n}^{\varepsilon}\right\|^{2}\right) \Gamma_{\varepsilon}\left(u_{n}^{\varepsilon}\right)+\int P_{\varepsilon}(x) \widehat{G}\left(\left|u_{n}^{\varepsilon}\right|\right)+\frac{1}{6} \int W_{\varepsilon}(x)\left|u_{n}^{\varepsilon}\right|^{3},
$$

consequently $\left|u_{n}^{\varepsilon}\right|_{\sigma} \leq d_{2}$ and $\left|u_{n}^{\varepsilon}\right|_{3} \leq d_{2}$. Similarly to (2.22) we get that

$$
\begin{aligned}
o(1)+\frac{1}{2}\left\|u_{n}^{\varepsilon}\right\|^{2} \leq & \varepsilon^{2} d_{3}+\eta\left(\left\|u_{n}^{\varepsilon}\right\|^{2}\right) \Gamma_{\varepsilon}^{\prime}\left(u_{n}^{\varepsilon}\right)\left(u_{n}^{\varepsilon+}-u_{n}^{\varepsilon-}\right) \\
& +\Re \int_{\left|u_{n}^{\varepsilon}\right| \geq r_{1}} P_{\varepsilon}(x) g\left(\left|u_{n}^{\varepsilon}\right|\right) u_{n}^{\varepsilon} \cdot \overline{u_{n}^{\varepsilon+}-u_{n}^{\varepsilon-}} \\
& +\Re \int W_{\varepsilon}(x)\left|u_{n}^{\varepsilon}\right| u_{n}^{\varepsilon} \cdot \overline{u_{n}^{\varepsilon+}-u_{n}^{\varepsilon-}}
\end{aligned}
$$

which, together with (2.23) and (2.24), implies either $\left\|u_{n}^{\varepsilon}\right\| \leq 1$ or as (2.25)

$$
\left\|u_{n}^{\varepsilon}\right\|^{2} \leq \varepsilon^{2} d_{3}+M_{1}\left\|u_{n}^{\varepsilon}\right\|+M_{2}\left\|u_{n}^{\varepsilon}\right\|^{1+\zeta}+M_{3},
$$

thus

$$
\left\|u_{n}^{\varepsilon}\right\| \leq \varepsilon^{2} d_{4}+\Lambda
$$

The proof is complete.

Based on this lemma, to prove our main results, it suffices to study $\widetilde{\Phi}_{\varepsilon}$ and get its critical points with critical values in $\left[0, \gamma_{b}\right]$. This will be done via a series of arguments. The first is to introduce the minimax values of $\widetilde{\Phi}_{\varepsilon}$. It is easy to verify the following lemma.

Lemma 3.13. $\widetilde{\Phi}_{\varepsilon}$ possesses the linking structure and the constants in Lemma 2.3 are true for $\widetilde{\Phi}_{\varepsilon}$. In addition, $\max _{v \in E_{e_{0}}} \widetilde{\Phi}_{\varepsilon}(v) \leq \gamma_{\mathrm{b}}$.

Define (see $[8,27])$

$$
c_{\varepsilon}:=\inf _{e \in E^{+} \backslash\{0\}} \max _{u \in E_{e}} \widetilde{\Phi}_{\varepsilon}(u) .
$$

As a consequence of Lemma 3.13 we have 
Lemma 3.14. $\tau \leq c_{\varepsilon} \leq \gamma_{\mathrm{b}}$.

We now describe further the minimax value $c_{\varepsilon}$. As before, for a fixed $u \in E^{+}$we define $\phi_{u}: E^{-} \rightarrow \mathbb{R}$ by

$$
\phi_{u}(v)=\widetilde{\Phi}_{\varepsilon}(u+v) .
$$

A direct computation gives, for any $v, z \in E^{-}$,

$$
\begin{aligned}
\phi_{u}^{\prime \prime}(v)[z, z] & =-\|z\|^{2}-\mathscr{F}_{\varepsilon}^{\prime \prime}(u+v)[z, z]-\Psi_{\varepsilon}^{\prime \prime}(u+v)[z, z], \\
& \leq-\|z\|^{2}-\mathscr{F}_{\varepsilon}^{\prime \prime}(u+v)[z, z],
\end{aligned}
$$

and

$$
\begin{aligned}
& \mathscr{F}_{\varepsilon}^{\prime \prime}(u+v)[z, z] \\
= & \left(4 \eta^{\prime \prime}\left(\|u+v\|^{2}\right)|\langle u+v, z\rangle|^{2}+2 \eta^{\prime}\left(\|u+v\|^{2}\right)\|z\|^{2}\right) \Gamma_{\varepsilon}(u+v) \\
& +4 \eta^{\prime}\left(\|u+v\|^{2}\right)\langle u+v, z\rangle \Gamma_{\varepsilon}^{\prime}(u+v) z \\
& +\eta\left(\|u+v\|^{2}\right) \Gamma_{\varepsilon}^{\prime \prime}(u+v)[z, z] .
\end{aligned}
$$

Combining (2.9)-(2.11) yields that there is $\varepsilon_{0} \in\left(0, \varepsilon_{1}\right]$ such that

$$
\phi_{u}^{\prime \prime}(v)[z, z] \leq-\frac{1}{2}\|z\|^{2} \quad \text { if } 0<\varepsilon \leq \varepsilon_{0} .
$$

Since

$$
\phi_{u}(v) \leq \frac{1}{2}\|u\|^{2}-\frac{1}{2}\|v\|^{2},
$$

there is $h_{\varepsilon}: E^{+} \rightarrow E^{-}$, uniquely defined, such that

$$
\phi_{u}\left(h_{\varepsilon}(u)\right)=\max _{v \in E^{-}} \phi_{u}(v)
$$

and

$$
v \neq h_{\varepsilon}(u) \Leftrightarrow \widetilde{\Phi}_{\varepsilon}(u+v)<\widetilde{\Phi}_{\varepsilon}\left(u+h_{\varepsilon}(u)\right) .
$$

It is clear that, for all $v \in E^{-}$

$$
0=\phi_{u}^{\prime}\left(h_{\varepsilon}(u)\right) v=-\left\langle h_{\varepsilon}(u), v\right\rangle-\mathscr{F}_{\varepsilon}^{\prime}\left(u+h_{\varepsilon}(u)\right) v-\Psi_{\varepsilon}^{\prime}\left(u+h_{\varepsilon}(u)\right) v .
$$

Observe that, similar to (3.4), we have for $u \in E^{+}$and $v \in E^{-}$

$$
\begin{aligned}
& \widetilde{\Phi}_{\varepsilon}\left(u+h_{\varepsilon}(u)\right)-\widetilde{\Phi}_{\varepsilon}(u+v) \\
= & \int_{0}^{1}(1-t)\left[\mathscr{F}_{\varepsilon}^{\prime \prime}\left(u+h_{\varepsilon}(u)+t\left(v-h_{\varepsilon}(u)\right)\right)\left[v-h_{\varepsilon}(u), v-h_{\varepsilon}(u)\right]\right. \\
& \left.+\Psi_{\varepsilon}^{\prime \prime}\left(u+h_{\varepsilon}(u)+t\left(v-h_{\varepsilon}(u)\right)\right)\left[v-h_{\varepsilon}(u), v-h_{\varepsilon}(u)\right]\right] d t \\
& +\frac{1}{2}\left\|v-h_{\varepsilon}(u)\right\|^{2} .
\end{aligned}
$$


Define $I_{\varepsilon}: E^{+} \rightarrow \mathbb{R}$ by

$$
I_{\varepsilon}(u)=\widetilde{\Phi}_{\varepsilon}\left(u+h_{\varepsilon}(u)\right)
$$

and set

$$
\mathscr{N}_{\varepsilon}:=\left\{u \in E^{+} \backslash\{0\}: I_{\varepsilon}^{\prime}(u) u=0\right\} .
$$

Lemma 3.15. For any $u \in E^{+} \backslash\{0\}$, there is a unique $t=t(u)>0$ such that $t u \in \mathscr{N}_{\varepsilon}$.

Proof. See $[2,14]$.

Lemma 3.16. $c_{\varepsilon}=\inf _{u \in \mathscr{N}_{\varepsilon}} I_{\varepsilon}(u)$.

Proof. Indeed, denoting $d_{\varepsilon}=\inf _{u \in \mathscr{N}_{\varepsilon}} I_{\varepsilon}(u)$, given $\underset{\widetilde{\Phi}}{e} \in E^{+}$, if $u=v+s e \in E_{e}$ with $\widetilde{\Phi}_{\varepsilon}(u)=\max _{z \in E_{e}} \widetilde{\Phi}_{\varepsilon}(z)$ then the restriction $\left.\widetilde{\Phi}_{\varepsilon}\right|_{E_{e}}$ of $\widetilde{\Phi}_{\varepsilon}$ on $E_{e}$ satisfies $\left(\left.\widetilde{\Phi}_{\varepsilon}\right|_{E_{e}}\right)^{\prime}(u)=0$ which implies $v=h_{\varepsilon}(s e)$ and $I_{\varepsilon}^{\prime}(s e)(s e)=0$, i.e. $\widetilde{\Phi}_{\varepsilon} \in \mathscr{N}_{\varepsilon}$. Thus $d_{\varepsilon} \leq c_{\varepsilon}$. While, on the other hand, if $w \in \mathscr{N}_{\varepsilon}$ then $\left(\left.\widetilde{\Phi}_{\varepsilon}\right|_{E_{w}}\right)^{\prime}(w+$ $\left.h_{\varepsilon}(w)\right)=0$, hence, $c_{\varepsilon} \leq \max _{u \in E_{w}} \widetilde{\Phi}_{\varepsilon}(u)=I_{\varepsilon}(w)$. Thus $d_{\varepsilon} \geq c_{\varepsilon}$.

Lemma 3.17. For any $e \in E^{+} \backslash\{0\}$, there is $T_{e}>0$ independent of $\varepsilon$ such that $t_{\varepsilon} \leq T_{e}$ for $t_{\varepsilon}>0$ satisfying $t_{\varepsilon} e \in \mathscr{N}_{\varepsilon}$.

Proof. Since $I_{\varepsilon}^{\prime}\left(t_{\varepsilon} e\right)\left(t_{\varepsilon} e\right)=0$, one get

$$
\widetilde{\Phi}_{\varepsilon}\left(t_{\varepsilon} e+h_{\varepsilon}\left(t_{\varepsilon} e\right)\right)=\max _{w \in E_{e}} \widetilde{\Phi}_{\varepsilon}(w) \geq \tau .
$$

This, together with Lemma 3.13, shows the assertion.

Next we estimate the regularities of critical points of $\widetilde{\Phi}_{\varepsilon}$. Let $\mathscr{K}_{\varepsilon}:=\{u \in$ $\left.E: \widetilde{\Phi}_{\varepsilon}^{\prime}(u)=0\right\}$ be the critical set of $\widetilde{\Phi}_{\varepsilon}$. It is easy to see that if $\mathscr{K}_{\varepsilon} \backslash\{0\} \neq \emptyset$ then $c_{\varepsilon}=\inf \left\{\widetilde{\Phi}_{\varepsilon}(u): u \in \mathscr{K}_{\varepsilon} \backslash\{0\}\right\}$ (see an argument of [14]).

For the subcritical case, using the same iterative argument of [15] one obtains easily the following

Lemma 3.18. If $u \in \mathscr{K}_{\varepsilon}$ with $\left|\widetilde{\Phi}_{\varepsilon}(u)\right| \leq C_{1}$, then, for any $q \in[2,+\infty)$, $u \in W^{1, q}\left(\mathbb{R}^{3}, \mathbb{C}^{4}\right)$ with $\|u\|_{W^{1, q}} \leq \Lambda_{q}$ where $\Lambda_{q}$ depends only on $C_{1}$ and $q$.

Proof. See [15]. We outline the proof as follows. From (2.8), we write

$$
\begin{aligned}
u & =H_{0}^{-1}\left(Q_{\varepsilon}(x) A_{\varepsilon, u}^{0} u-\sum_{k=1}^{3} Q_{\varepsilon}(x) \alpha_{k} A_{\varepsilon, u}^{k} u+\sum_{j=1}^{J} W_{j}(\varepsilon x)|u|^{p_{j}-2} u\right) \\
& =u_{1}+u_{2}
\end{aligned}
$$

where

$$
u_{1}=H_{0}^{-1}\left(\sum_{j=1}^{J} W_{j}(\varepsilon x)|u|^{p_{j}-2} u\right)
$$


and

$$
u_{2}=H_{0}^{-1}\left(Q_{\varepsilon} \cdot A_{\varepsilon, u}^{0} u-\sum_{k=1}^{3} Q_{\varepsilon} \cdot \alpha_{k} A_{\varepsilon, u}^{k} u\right) .
$$

By Hölder's inequality, for $q \geq 2$

$$
\left|Q_{\varepsilon} \alpha_{k} A_{\varepsilon, u}^{k} u\right|_{s} \leq|Q|_{\infty}\left|A_{\varepsilon, u}^{k}\right|_{6}|u|_{q}
$$

with $\frac{1}{s}=\frac{1}{6}+\frac{1}{q}$ and

$$
\left.\left.\left|W_{j \varepsilon} \cdot\right| u\right|^{p_{j}-2} u\right|_{t} \leq\left|W_{j}\right|_{\infty}|u|_{t\left(p_{j}-1\right)}^{p_{j}-1} .
$$

Thus, the standard argument shows that $u \in \cap_{q \geq 2} L^{q}$, therefore, $u_{1} \in$ $\cap_{q \geq 2} W^{1, q}$ an $u_{2} \in \cap_{6>q \geq 2} W^{1, q}$.

By Sobolev embedding theorems, $u \in C^{0, \vartheta}$ for some $\vartheta \in(0,1)$. This together with elliptic regularity (see [18]), shows $A_{\varepsilon, u}^{k} \in W_{l o c}^{2,6}\left(\mathbb{R}^{3}\right) \cap L^{6}\left(\mathbb{R}^{3}\right.$ ) for $k=0,1,2,3$ and

$$
\left\|A_{\varepsilon, u}^{k}\right\|_{W^{2,6}\left(B_{1}(x)\right)} \leq C_{2}\left(\varepsilon^{2}|Q|_{\infty}|u|_{L^{12}\left(B_{2}(x)\right)}^{2}+\left|A_{\varepsilon, u}^{k}\right|_{L^{6}\left(B_{2}(x)\right)}\right)
$$

for all $x \in \mathbb{R}^{3}$, with $C_{2}$ independent of $x$ and $\varepsilon$, where $B_{r}(x)=\left\{y \in \mathbb{R}^{3}\right.$ : $|y-x|<r\}$ for $r>0$. Since $W^{2,6}\left(B_{1}(x)\right) \hookrightarrow C^{1}\left(B_{1}(x)\right)$, we have

$$
\left\|A_{\varepsilon, u}^{k}\right\|_{C^{1}\left(B_{1}(x)\right)} \leq C_{3}\left(\varepsilon^{2}|Q|_{\infty}|u|_{L^{12}\left(B_{2}(x)\right)}^{2}+\left|A_{\varepsilon, u}^{k}\right|_{L^{6}\left(B_{2}(x)\right)}\right)
$$

for all $x \in \mathbb{R}^{3}$ with $C_{3}$ independent of $x$ and $\varepsilon$. Consequently $A_{\varepsilon, u}^{k} \in L^{\infty}$ and that yields

$$
\left|Q_{\varepsilon} \alpha_{k} A_{\varepsilon, u}^{k} u\right|_{s} \leq|Q|_{\infty}\left|A_{\varepsilon, u}^{k}\right|_{\infty}|u|_{s} .
$$

Thus $u_{2} \in \cap_{q \geq 2} W^{1, q}$, and combining with $u_{1} \in \cap_{q \geq 2} W^{1, q}$, the conclusion is obtained.

Concerning the critical nonlinearity, the standard bootstrap argument does not yield regularity of finite action weak solutions (see [5, 6]). Motivated by Takeshi Isobe [28], we give the following lemma

Lemma 3.19. Let $u$ be a weak solution to the critical system in $E$. Then $u \in \cap_{q \geq 2} W_{l o c}^{1, q}\left(\mathbb{R}^{3}\right) \cap L^{\infty}\left(\mathbb{R}^{3}\right)$.

Proof. Recall that for $x \in \mathbb{R}^{3}$ being arbitrary, by elliptic regularity (see [18]), for $k=0,1,2,3$, we infer

$$
\left\|A_{\varepsilon, u}^{k}\right\|_{W^{2, \frac{3}{2}\left(B_{2}(x)\right)}} \leq C\left(\left|A_{\varepsilon, u}^{k}\right|_{L^{\frac{3}{2}\left(B_{3}(x)\right)}}+\varepsilon^{2}|Q|_{\infty}|u|_{3}^{2}\right)
$$


with $C$ independent of $x, u$ and $\varepsilon$. And since $A_{\varepsilon, u}^{k} \in L^{6}\left(\mathbb{R}^{3}\right)$, one has

$$
\left\|A_{\varepsilon, u}^{k}\right\|_{W^{2, \frac{3}{2}\left(B_{2}(x)\right)}} \leq C\left(3 \sqrt{\pi}\left|A_{\varepsilon, u}^{k}\right|_{L^{6}\left(B_{3}(x)\right)}+\varepsilon^{2}|Q|_{\infty}|u|_{3}^{2}\right) .
$$

Set $x \in \mathbb{R}^{3}$ fixed, let $\bar{\rho} \in C_{c}^{\infty}\left(B_{2}(x)\right)$ be arbitrary. Take $\bar{\eta} \in C_{c}^{\infty}\left(B_{2}(x)\right)$ such that $\bar{\eta}=1$ on supp $\bar{\rho}$. We deduce, letting $D=i \alpha \cdot \nabla$,

$$
\begin{aligned}
D(\bar{\rho} u) & =\bar{\rho} D u+D \bar{\rho} \cdot u \\
& =\bar{\eta} \cdot \bar{\rho} D u+D \bar{\rho} \cdot u .
\end{aligned}
$$

Noting that

$$
D u=a \beta u+\omega u+Q_{\varepsilon} \cdot A_{\varepsilon, u}^{0} u-\sum_{k=1}^{3} Q_{\varepsilon} \cdot \alpha_{k} A_{\varepsilon, u}^{k} u+P_{\varepsilon} \cdot g(|u|) u+W_{\varepsilon} \cdot|u| u,
$$

one has

$$
D \bar{\rho} \cdot u=H_{0}(\bar{\rho} u)-T_{\varepsilon, u}(\bar{\rho} u),
$$

where, for $1<s<3, T_{\varepsilon, u}: W^{1, s}\left(B_{2}(x)\right) \rightarrow L^{s}\left(B_{2}(x)\right)$ is defined by

$$
w \mapsto \bar{\eta} \cdot\left[Q_{\varepsilon} \cdot A_{\varepsilon, u}^{0}-\sum_{k=1}^{3} Q_{\varepsilon} \cdot \alpha_{k} A_{\varepsilon, u}^{k}+P_{\varepsilon} \cdot g(|u|)+W_{\varepsilon} \cdot|u|\right] w .
$$

Notice that, by the Sobolev embedding $W^{1, s}\left(B_{2}(x)\right) \hookrightarrow L^{\frac{3 s}{3-s}}\left(B_{2}(x)\right)$ and Hölder inequality, we have $T_{\varepsilon, u}(w) \in L^{s}\left(B_{2}(x)\right)$ for $w \in W^{1, s}\left(B_{2}(x)\right)$ and the above map is well defined. Using Minkowski and Hölder inequalities, the operator norm can be estimated as

$$
\left\|T_{\varepsilon, u}\right\|_{W^{1, s} \rightarrow L^{s}} \leq C_{1}\left(|u|_{L^{3}(B)}+|B|^{\frac{s}{3}}+\sum_{k=0}^{3}\left|A_{\varepsilon, u}^{k}\right|_{L^{6}(B)}\right)
$$

for some constant $C_{1}$ (depending on $s$ ), where $B:=\operatorname{supp} \bar{\eta}$.

Since $0 \notin \sigma\left(H_{0}\right)$,

$$
H_{0}-T_{\varepsilon, u}: W^{1, s}\left(B_{2}(x)\right) \rightarrow L^{s}\left(B_{2}(x)\right)
$$

is invertible if $|B|$ is small.

Therefore, by (3.11), there is a unique solution $w \in W^{1, s}\left(B_{2}(x)\right)$ to the equation

$$
H_{0} w-T_{\varepsilon, u}(w)=D \bar{\rho} \cdot u \quad \text { in } B_{2}(x) .
$$

On the other hand, we have a well defined map

$$
T_{\varepsilon, u}: L^{3}\left(B_{2}(x)\right) \rightarrow W^{-1,3}\left(B_{2}(x)\right) .
$$


Indeed, by Hölder inequality, we have $T_{\varepsilon, u}(w) \in L^{\frac{3}{2}}\left(B_{2}(x)\right)$. Taking into account that $L^{\frac{3}{2}}\left(B_{2}(x)\right) \subset W^{-1,3}\left(B_{2}(x)\right)$ by the Sobolev embedding, the above map is well defined and the operator norm is estimated as before:

$$
\left\|T_{\varepsilon, u}\right\|_{L^{3} \rightarrow W^{-1,3}} \leq C_{2}\left(|B|^{\frac{1}{3}}+|u|_{L^{3}(B)}+\sum_{k=0}^{3}\left|A_{\varepsilon, u}^{k}\right|_{L^{6}(B)}\right) .
$$

Thus,

$$
H_{0}-T_{\varepsilon, u}: L^{3}\left(B_{2}(x)\right) \rightarrow W^{-1,3}\left(B_{2}(x)\right)
$$

is invertible if $|B|$ is small and there is a unique solution $\tilde{w} \in L^{3}\left(B_{2}(x)\right)$ to the equation

$$
H_{0} \tilde{w}-T_{\varepsilon, u}(\tilde{w})=D \bar{\rho} \cdot u \text {. }
$$

Consequently, $\tilde{w}=\bar{\rho} u$ by (3.11). On the other hand, with the fact that $W^{1, s}\left(B_{2}(x)\right) \hookrightarrow L^{3}\left(B_{2}(x)\right)$ if $3 / 2 \leq s<3, w \in W^{1, s}$ is also a $L^{3}$ solution to (3.12) provided $3 / 2 \leq s<3$. Thus, by uniqueness, $w=\bar{\rho} u$ and $\bar{\rho} u \in W^{1, s}\left(B_{2}(x)\right)$ for any $s \in[3 / 2,3)$ provided $B=\operatorname{supp} \bar{\eta}$ is small. Since $\bar{\rho}$ and $\bar{\eta}$ arbitrary, one has $u \in W^{1, s}\left(B_{1}(x)\right)$ for any $s \in[3 / 2,3)$.

Therefore, by Sobolev embedding, we obtain $u \in \cap_{q \geq 2} L_{l o c}^{q}\left(\mathbb{R}^{3}\right)$ and this implies $u \in \cap_{q \geq 2} W_{l o c}^{1, q}\left(\mathbb{R}^{3}\right)$. Finally, with the elliptic estimate, we obtain $u \in L^{\infty}$.

Remark 3.20. Let $\mathscr{L}_{\varepsilon}$ denote the set of all least energy solutions of $\widetilde{\Phi}_{\varepsilon}$. If $u \in \mathscr{L}_{\varepsilon}, \widetilde{\Phi}_{\varepsilon}(u)=c_{\varepsilon} \leq \gamma_{\mathfrak{b}}$. Recall that $\mathscr{L}_{\varepsilon}$ is bounded in $E$ with upper bound $\Lambda$ independent of $\varepsilon$. As a consequence of Lemmas 3.18 and 3.19, together with the Sobolev embedding theorem, we see that there is $C_{\infty}>0$ independent of $\varepsilon$ with

$$
\|u\|_{\infty} \leq C_{\infty} \quad \text { for all } u \in \mathscr{L}_{\varepsilon} .
$$

\section{Proof of the main result}

Throughout this section we suppose $\left(Q_{0}\right)$ and $\left(P_{0}\right)-\left(P_{1}\right)$ are satisfied for the subcritical case and $\left(g_{1}\right)-\left(g_{2}\right),\left(Q_{0}\right)$ and $\left(P_{2}\right)-\left(P_{3}\right)$ are satisfied for the critical case, and recall that we always assume 0 in $\mathscr{P}$ and $\mathscr{W}$. The proof of the main theorem will be finished in three parts: Existence, Concentration, and Exponential decay.

\section{Part 1. Existence}

Its proof is carried out in three lemmas. The modified problem gives us an access to Lemma 4.1, which is the key ingredient for Lemma 4.2. 
In the sequel, denoted by $a_{1}=W_{1}(0), \cdots, a_{J}=W_{J}(0), \mu=P(0)$ and $\chi=W(0)$, we remark that

$$
\begin{aligned}
& W_{j}(\varepsilon x) \rightarrow a_{j} \quad j=1, \cdots, J \\
& P(\varepsilon x) \rightarrow \mu \\
& W(\varepsilon x) \rightarrow \chi
\end{aligned}
$$

uniformly on bounded sets of $x$ as $\varepsilon \rightarrow 0$. Setting $\vec{a}=\left(a_{1}, \cdots, a_{J}\right), \vec{m}=$ $\left(m_{1}, \cdots, m_{J}\right)$, let $\mathscr{T}_{\infty}$ and $\overline{\mathscr{T}}_{\infty}$ be the functionals defined by

$$
\mathscr{T}_{\infty}(u)=\left\{\begin{array}{l}
\mathscr{T}_{\vec{m}}(u) \text { for the subcritical case; } \\
\mathscr{T}_{m l}^{*}(u) \text { for the critical case }
\end{array}\right.
$$

and

$$
\overline{\mathscr{T}}_{\infty}(u)=\left\{\begin{array}{l}
\mathscr{T}_{\vec{a}}(u) \text { for the subcritical case; } \\
\mathscr{T}_{\mu \chi}^{*}(u) \text { for the critical case }
\end{array}\right.
$$

and $J_{\infty}, \bar{J}_{\infty}$ denote the associated reduction functionals on $E^{+}$. Correspondingly, denote

$$
\gamma_{\infty}=\left\{\begin{array}{l}
\gamma_{\vec{m}} \text { for the subcritical case } \\
\gamma_{m l}^{*} \text { for the critical case }
\end{array}\right.
$$

and

$$
\bar{\gamma}_{\infty}=\left\{\begin{array}{l}
\gamma_{\vec{a}} \text { for the subcritical case } \\
\gamma_{\mu \chi}^{*} \text { for the critical case. }
\end{array}\right.
$$

For notation convenience, we also denote

$$
\mathscr{O}=\left\{\begin{array}{l}
\mathscr{W}_{0} \text { for the subcritical case } \\
\mathscr{P} \text { for the critical case. }
\end{array}\right.
$$

And our existence results are organized as

Lemma 4.1. $\lim \sup _{\varepsilon \rightarrow 0} c_{\varepsilon} \leq \bar{\gamma}_{\infty}$. In particular, if $\mathscr{O} \neq \emptyset$,

$$
\lim _{\varepsilon \rightarrow 0} c_{\varepsilon}=\bar{\gamma}_{\infty}=\gamma_{\infty} .
$$

Lemma 4.2. $c_{\varepsilon}$ is attained for all small $\varepsilon>0$.

Lemma 4.3. $\mathscr{L}_{\varepsilon}$ is compact for all small $\varepsilon>0$ in $H^{1}$. 
Proof of Lemma 4.1. Firstly we show that

$$
\liminf _{\varepsilon \rightarrow 0} c_{\varepsilon} \geq \gamma_{\infty}
$$

Arguing indirectly, assume that $\liminf _{\varepsilon \rightarrow 0} c_{\varepsilon}<\gamma_{\infty}$. By definition and Lemma 3.16 we can choose $e_{j} \in \mathscr{N}_{\varepsilon}$ and $\delta>0$ such that

$$
\max _{u \in E_{e_{j}}} \widetilde{\Phi}_{\varepsilon_{j}}(u) \leq \gamma_{\infty}-\delta
$$

as $\varepsilon_{j} \rightarrow 0$. Since $\left(P_{0}\right),\left(P_{2}\right)$ and $\mathscr{F}(u)=o(1)$ as $\varepsilon \rightarrow 0$ uniformly in $u$ (by (2.9) and the definition of $\eta$ ), the representations of $\widetilde{\Phi}_{\varepsilon}$ and $\mathscr{T}_{\infty}$ imply that $\widetilde{\Phi}_{\varepsilon}(u) \geq \mathscr{T}_{\infty}(u)-\delta / 2$ for all $u \in E$ and $\varepsilon$ small. Note also that $\gamma_{\infty} \leq J_{\infty}\left(e_{j}\right) \leq \max _{u \in E_{e_{j}}} \mathscr{T}_{\infty}(u)$. Therefore we get, for all $\varepsilon_{j}$ small,

$$
\gamma_{\infty}-\delta \geq \max _{u \in E_{e_{j}}} \widetilde{\Phi}_{\varepsilon_{j}}(u) \geq \max _{u \in E_{e_{j}}} \mathscr{T}_{\infty}(u)-\frac{\delta}{2} \geq \gamma_{\infty}-\frac{\delta}{2}
$$

a contradiction.

We now turn to prove the desired conclusion. We only check the critical case because the subcritical case can be treated similarly.

Set $P^{0}(x)=\mu-P(x), W^{0}(x)=\chi-W(x)$ and $P_{\varepsilon}^{0}(x)=P^{0}(\varepsilon x), W_{\varepsilon}^{0}(x)=$ $W^{0}(\varepsilon x)$. Then

$$
\widetilde{\Phi}_{\varepsilon}(u)=\mathscr{T}_{\mu \chi}^{*}(u)-\mathscr{F}_{\varepsilon}(u)+\int P_{\varepsilon}^{0}(x) G(|u|)+\frac{1}{3} \int W_{\varepsilon}^{0}|u|^{3} .
$$

In virtue of Lemma 3.8, let $u=u^{+}+u^{-} \in \mathscr{R}_{\mu \chi}^{*}$ and set $e=u^{+}$. Surely, $e \in \mathscr{M}_{\mu \chi}^{*}, \mathscr{J}_{\mu \chi}^{*}(e)=u^{-}$and $J_{\mu \chi}^{*}(e)=\gamma_{\mu \chi}^{*}$. There is a unique $t_{\varepsilon}>0$ such that $t_{\varepsilon} e \in \mathscr{N}_{\varepsilon}$ and one has

$$
c_{\varepsilon} \leq I_{\varepsilon}\left(t_{\varepsilon} e\right) .
$$

By Lemma $3.17 t_{\varepsilon}$ is bounded. Hence, without loss of generality we can assume $t_{\varepsilon} \rightarrow t_{0}$ as $\varepsilon \rightarrow 0$. By using (3.4) and (3.9), we infer

$$
\begin{aligned}
& \frac{1}{2}\left\|v_{\varepsilon}\right\|^{2}+(I)=\widetilde{\Phi}_{\varepsilon}\left(w_{\varepsilon}\right)-\widetilde{\Phi}_{\varepsilon}\left(u_{\varepsilon}\right) \\
= & \mathscr{T}_{\mu \chi}^{*}\left(w_{\varepsilon}\right)-\mathscr{T}_{\mu \chi}^{*}\left(u_{\varepsilon}\right)-\mathscr{F}_{\varepsilon}\left(w_{\varepsilon}\right)+\mathscr{F}_{\varepsilon}\left(u_{\varepsilon}\right) \\
& +\int P_{\varepsilon}^{0}(x)\left(G\left(\left|w_{\varepsilon}\right|\right)-G\left(\left|u_{\varepsilon}\right|\right)\right) \\
& +\frac{1}{3} \int W_{\varepsilon}^{0}(x)\left(\left|w_{\varepsilon}\right|^{3}-\left|u_{\varepsilon}\right|^{3}\right)
\end{aligned}
$$

where, setting

$$
u_{\varepsilon}=t_{\varepsilon} e+\mathscr{J}_{\mu \chi}^{*}\left(t_{\varepsilon} e\right), w_{\varepsilon}=t_{\varepsilon} e+h_{\varepsilon}\left(t_{\varepsilon} e\right), v_{\varepsilon}=u_{\varepsilon}-w_{\varepsilon},
$$




$$
(I):=\int_{0}^{1}(1-s)\left(\mathscr{F}_{\varepsilon}^{\prime \prime}\left(w_{\varepsilon}+s v_{\varepsilon}\right)\left[v_{\varepsilon}, v_{\varepsilon}\right]+\Psi_{\varepsilon}^{\prime \prime}\left(w_{\varepsilon}+s v_{\varepsilon}\right)\left[v_{\varepsilon}, v_{\varepsilon}\right]\right) d s .
$$

Taking into account that

$$
\mathscr{F}_{\varepsilon}\left(u_{\varepsilon}\right)-\mathscr{F}_{\varepsilon}\left(w_{\varepsilon}\right)=\mathscr{F}_{\varepsilon}^{\prime}\left(w_{\varepsilon}\right) v_{\varepsilon}+\int_{0}^{1}(1-s) \mathscr{F}_{\varepsilon}^{\prime \prime}\left(w_{\varepsilon}+s v_{\varepsilon}\right)\left[v_{\varepsilon}, v_{\varepsilon}\right] d s
$$

and

$$
\begin{aligned}
& \int P_{\varepsilon}^{0}(x)\left(G\left(\left|w_{\varepsilon}\right|\right)-G\left(\left|u_{\varepsilon}\right|\right)\right)+\frac{1}{3} \int W_{\varepsilon}^{0}\left(\left|w_{\varepsilon}\right|^{3}-\left|u_{\varepsilon}\right|^{3}\right) \\
= & -\int P_{\varepsilon}^{0}(x) g\left(\left|u_{\varepsilon}\right|\right) u_{\varepsilon} \cdot \overline{v_{\varepsilon}}-\int W_{\varepsilon}^{0}(x)\left|u_{\varepsilon}\right| u_{\varepsilon} \cdot \overline{v_{\varepsilon}} \\
& +\int_{0}^{1}(1-s) \mathscr{G}_{m l}^{* \prime \prime}\left(u_{\varepsilon}-s v_{\varepsilon}\right)\left[v_{\varepsilon}, v_{\varepsilon}\right] d s \\
& -\int_{0}^{1}(1-s) \Psi_{\varepsilon}^{\prime \prime}\left(u_{\varepsilon}-s v_{\varepsilon}\right)\left[v_{\varepsilon}, v_{\varepsilon}\right] d s,
\end{aligned}
$$

setting

$$
(I I):=\int_{0}^{1}(1-s) \Psi_{\varepsilon}^{\prime \prime}\left(u_{\varepsilon}-s v_{\varepsilon}\right)\left[v_{\varepsilon}, v_{\varepsilon}\right] d s,
$$

by (3.8) one has

$$
\begin{aligned}
& \frac{1}{2}\left\|v_{\varepsilon}\right\|^{2}+(I)+(I I) \\
\leq & \mathscr{F}_{\varepsilon}^{\prime}\left(w_{\varepsilon}\right) v_{\varepsilon}+\int_{0}^{1}(1-s) \mathscr{F}_{\varepsilon}^{\prime \prime}\left(w_{\varepsilon}+s v_{\varepsilon}\right)\left[v_{\varepsilon}, v_{\varepsilon}\right] d s \\
& -\int P_{\varepsilon}^{0}(x) g\left(\left|u_{\varepsilon}\right|\right) u_{\varepsilon} \cdot \overline{v_{\varepsilon}}-\int W_{\varepsilon}^{0}(x)\left|u_{\varepsilon}\right| u_{\varepsilon} \cdot \overline{v_{\varepsilon}} .
\end{aligned}
$$

So we deduce, noticing that $0 \leq P_{\varepsilon}^{0}(x)$ and $0 \leq W_{\varepsilon}^{0}(x)$,

$$
\begin{aligned}
& \frac{1}{2}\left\|v_{\varepsilon}\right\|^{2}+\int_{0}^{1}(1-s) \Psi_{\varepsilon}^{\prime \prime}\left(w_{\varepsilon}+s v_{\varepsilon}\right)\left[v_{\varepsilon}, v_{\varepsilon}\right] d s \\
\leq & \left|\mathscr{F}_{\varepsilon}^{\prime}\left(w_{\varepsilon}\right) v_{\varepsilon}\right|+\int P_{\varepsilon}^{0}(x) g\left(\left|u_{\varepsilon}\right|\right)\left|u_{\varepsilon}\right| \cdot\left|v_{\varepsilon}\right|+\int W_{\varepsilon}^{0}(x)\left|u_{\varepsilon}\right|^{2} \cdot\left|v_{\varepsilon}\right| .
\end{aligned}
$$

Since $t_{\varepsilon} \rightarrow t_{0}$, it is clear that $\left\{u_{\varepsilon}\right\},\left\{w_{\varepsilon}\right\}$ and $\left\{v_{\varepsilon}\right\}$ are bounded, hence, by the definitions and (2.9), (2.10),

$$
\mathscr{F}_{\varepsilon}\left(z_{\varepsilon}\right)=o(1), \quad\left\|\mathscr{F}_{\varepsilon}^{\prime}\left(z_{\varepsilon}\right)\right\|=o(1)
$$

as $\varepsilon \rightarrow 0$ for $z_{\varepsilon}=u_{\varepsilon}, w_{\varepsilon}, v_{\varepsilon}$. Moreover, by noting that for $q \in[2,3]$

$$
\limsup _{r \rightarrow \infty} \int_{|x|>r}\left|u_{\varepsilon}\right|^{q}=0
$$


use the assumption of $0 \in \mathscr{P}$ one deduces

$$
\begin{aligned}
& \int\left(W_{\varepsilon}^{0}(x)\right)^{3 / 2}\left|u_{\varepsilon}\right|^{3} \\
= & \left(\int_{|x| \leq r}+\int_{|x|>r}\right)\left(W_{\varepsilon}^{0}(x)\right)^{3 / 2}\left|u_{\varepsilon}\right|^{3} \\
\leq & \int_{|x| \leq r}\left(W_{\varepsilon}^{0}(x)\right)^{3 / 2}\left|u_{\varepsilon}\right|^{3}+l^{3 / 2} \int_{|x|>r}\left|u_{\varepsilon}\right|^{3} \\
= & o(1)
\end{aligned}
$$

and analogously, by $\left(g_{1}\right)$,

$$
\int P_{\varepsilon}^{0}(x) g\left(\left|u_{\varepsilon}\right|\right)\left|u_{\varepsilon}\right| \cdot\left|v_{\varepsilon}\right|=o(1)
$$

as $\varepsilon \rightarrow 0$. Thus by (4.7) one has $\left\|v_{\varepsilon}\right\|^{2} \rightarrow 0$, that is, $h_{\varepsilon}\left(t_{\varepsilon} e\right) \rightarrow \mathscr{J}_{\mu \chi}^{*}\left(t_{0} e\right)$. Consequently,

$$
\int P_{\varepsilon}^{0}(x) G\left(\left|w_{\varepsilon}\right|\right)+\frac{1}{3} \int W_{\varepsilon}^{0}(x)\left|w_{\varepsilon}\right|^{3} \rightarrow 0
$$

as $\varepsilon \rightarrow 0$. This, jointly with (4.5), we have

$$
\widetilde{\Phi}_{\varepsilon}\left(w_{\varepsilon}\right)=\mathscr{T}_{\mu \chi}^{*}\left(w_{\varepsilon}\right)+o(1)=\mathscr{T}_{\mu \chi}^{*}\left(u_{\varepsilon}\right)+o(1),
$$

that is,

$$
I_{\varepsilon}\left(t_{\varepsilon} e\right)=J_{\mu \chi}^{*}\left(t_{0} e\right)+o(1)
$$

as $\varepsilon \rightarrow 0$. Then, since

$$
J_{\mu \chi}^{*}\left(t_{0} e\right) \leq \max _{v \in E_{e}} \mathscr{T}_{\mu \chi}^{*}(v)=J_{\mu \chi}^{*}(e)=\gamma_{\mu \chi}^{*},
$$

we obtain by (4.6)

$$
\limsup _{\varepsilon \rightarrow 0} c_{\varepsilon} \leq \lim _{\varepsilon \rightarrow 0} I_{\varepsilon}\left(t_{\varepsilon} e\right)=J_{\mu \chi}^{*}\left(t_{0} e\right) \leq \gamma_{\mu \chi}^{*} .
$$

Remark that, for the subcritical case, one may replace $P(x), W(x), P^{0}(x)$ and $W^{0}(x)$ with $W_{j}(x)$ and $W_{j}^{0}(x)=a_{j}-W_{j}(x), j=1, \cdots, J$ respectively. With some obvious modifications, one gets $\lim \sup _{\varepsilon \rightarrow 0} c_{\varepsilon} \leq \gamma_{\vec{a}}$. Therefore, we proved

$$
\limsup _{\varepsilon \rightarrow 0} c_{\varepsilon} \leq \bar{\gamma}_{\infty}
$$

Suppose additionally $\mathscr{O} \neq \emptyset$ (that is $0 \in \mathscr{O}$ ), we find $\vec{a}=\vec{m}$ and $\mu=m$, $\chi=l$, then $\gamma_{\infty}=\bar{\gamma}_{\infty}$ and obviously, by (4.4), we have

$$
\lim _{\varepsilon \rightarrow 0} c_{\varepsilon}=\gamma_{\infty}=\bar{\gamma}_{\infty}
$$


Proof of Lemma 4.2. Given $\varepsilon>0$, let $\left\{u_{n}\right\} \subset \mathscr{N}_{\varepsilon}$ be a minimization sequence: $I_{\varepsilon}\left(u_{n}\right) \rightarrow c_{\varepsilon}$. By the Ekeland variational principle we can assume that $\left\{u_{n}\right\}$ is in fact a $(P S)_{c_{\varepsilon}}$-sequence for $I_{\varepsilon}$ on $E^{+}$(see $[22,30]$ ). Then $w_{n}=u_{n}+h_{\varepsilon}\left(u_{n}\right)$ is a $(P S)_{c_{\varepsilon}}$-sequence for $\widetilde{\Phi}_{\varepsilon}$ on $E$. It is clear that $\left\{w_{n}\right\}$ is bounded, hence is a $(C)_{c_{\varepsilon}}$-sequence. We can assume without loss of generality that $w_{n} \rightarrow w_{\varepsilon}=w_{\varepsilon}^{+}+w_{\varepsilon}^{-} \in \mathscr{K}_{\varepsilon}$ in $E$. If $w_{\varepsilon} \neq 0$ then $\widetilde{\Phi}_{\varepsilon}\left(w_{\varepsilon}\right)=c_{\varepsilon}$. So we are going to show that $w_{\varepsilon} \neq 0$ for all small $\varepsilon>0$.

For this end, take $\vec{\kappa}=\left(\kappa_{1}, \cdots, \kappa_{J}\right)>0$ and $\kappa>0$ satisfies respectively

$$
\left\{\begin{array}{l}
\limsup _{|x| \rightarrow \infty} W_{j_{0}}(x)<\kappa_{j_{0}}<\max _{\mathbb{R}^{3}} W_{j_{0}}, \\
k_{j}=W_{j}(0) \quad j \neq j_{0}, \\
\limsup _{|x| \rightarrow \infty} P(x)<\kappa<\max _{\mathbb{R}^{3}} P \quad \text { for the subcritical case }
\end{array}\right.
$$

and define

$$
\begin{aligned}
& W_{j}^{\kappa}(x)=\min \left\{\kappa_{j}, W_{j}(x)\right\} \text { for the subcritical case, } \\
& P^{\kappa}(x)=\min \{\kappa, P(x)\} \text { for the critical case. }
\end{aligned}
$$

For the later use, we set

$$
A=\left\{x \in \mathbb{R}^{3}: P(x)>\kappa \text { or } W_{j}(x)>\kappa_{j} \text { for } j=1, \cdots, J\right\}
$$

and $A_{\varepsilon}=\left\{x \in \mathbb{R}^{3}: \varepsilon x \in A\right\}$. Following $\left(P_{0}\right)$ and $\left(P_{2}\right), A_{\varepsilon}$ is a bounded set for any fixed $\varepsilon>0$. Note that, for the critical case, $\kappa>m_{\infty}$, so we infer

$$
\kappa^{-1} \cdot l^{\sigma-2}<\mathcal{R}_{\sigma} .
$$

And invoking Lemma 3.7, (4.8) implies $\gamma_{\kappa l}^{*}$ is attained.

Now we consider the functionals

$$
\widetilde{\Phi}_{\varepsilon}^{\kappa}(u)=\frac{1}{2}\left(\left\|u^{+}\right\|^{2}-\left\|u^{-}\right\|^{2}\right)-\mathscr{F}_{\varepsilon}(u)-\sum_{j=1}^{J} \frac{1}{p_{j}} \int_{\mathbb{R}^{3}} W_{j}^{\kappa}(\varepsilon x)|u|^{p_{j}}
$$

for the subcritical case and

$$
\widetilde{\Phi}_{\varepsilon}^{\kappa}(u)=\frac{1}{2}\left(\left\|u^{+}\right\|^{2}-\left\|u^{-}\right\|^{2}\right)-\mathscr{F}_{\varepsilon}(u)-\int P_{\varepsilon}^{\kappa}(x) G(|u|)-\frac{1}{3} \int W_{\varepsilon}(x)|u|^{3}
$$

for the critical case. As before define correspondingly $h_{\varepsilon}^{\kappa}: E^{+} \rightarrow E^{-}$, $I_{\varepsilon}^{\kappa}: E^{+} \rightarrow \mathbb{R}, \mathscr{N}_{\varepsilon}^{\kappa}, c_{\varepsilon}^{\kappa}$ and so on. As done in the proof of Lemma 4.1,

$$
\lim _{\varepsilon \rightarrow 0} c_{\varepsilon}^{\kappa}=\gamma_{\infty}^{\kappa}
$$

where

$$
\gamma_{\infty}^{\kappa}=\left\{\begin{array}{l}
\gamma_{\vec{k}} \text { for the subcritical case } \\
\gamma_{\kappa l}^{*} \text { for the critical case. }
\end{array}\right.
$$


Assume by contradiction that there is a sequence $\varepsilon_{j} \rightarrow 0$ with $w_{\varepsilon_{j}}=0$. Then $w_{n}=u_{n}+h_{\varepsilon_{j}}\left(u_{n}\right) \rightarrow 0$ in $E, u_{n} \rightarrow 0$ in $L_{l o c}^{q}$ for $q \in[1,3)$, and $w_{n}(x) \rightarrow 0$ a.e. in $x \in \mathbb{R}^{3}$. Let $t_{n}>0$ be such that $t_{n} u_{n} \in \mathscr{N}_{\varepsilon_{j}}^{\kappa}$. Then $\left\{t_{n}\right\}$ is bounded and one may assume $t_{n} \rightarrow t_{0}$ as $n \rightarrow \infty$. Remark that $h_{\varepsilon_{j}}^{\kappa}\left(t_{n} u_{n}\right) \rightarrow 0$ in $E$ and $h_{\varepsilon_{j}}^{\kappa}\left(t_{n} u_{n}\right) \rightarrow 0$ in $L_{l o c}^{q}$ for $q \in[1,3)$ as $n \rightarrow \infty$ (see $[2])$. Moreover, $\widetilde{\Phi}_{\varepsilon_{j}}\left(t_{n} u_{n}+h_{\varepsilon_{j}}^{\kappa}\left(t_{n} u_{n}\right)\right) \leq I_{\varepsilon_{j}}\left(u_{n}\right)$. We obtain, for the critical case,

$$
\begin{aligned}
c_{\varepsilon_{j}}^{\kappa} & \leq I_{\varepsilon_{j}}^{\kappa}\left(t_{n} u_{n}\right)=\widetilde{\Phi}_{\varepsilon_{j}}^{\kappa}\left(t_{n} u_{n}+h_{\varepsilon_{j}}^{\kappa}\left(t_{n} u_{n}\right)\right) \\
& =\widetilde{\Phi}_{\varepsilon_{j}}\left(t_{n} u_{n}+h_{\varepsilon_{j}}^{\kappa}\left(t_{n} u_{n}\right)\right)+\int\left(P_{\varepsilon_{j}}(x)-P_{\varepsilon_{j}}^{\kappa}(x)\right) G\left(\left|t_{n} u_{n}+h_{\varepsilon_{j}}^{\kappa}\left(t_{n} u_{n}\right)\right|\right) \\
& \leq I_{\varepsilon_{j}}\left(u_{n}\right)+\int_{A_{\varepsilon_{j}}}\left(P_{\varepsilon_{j}}(x)-P_{\varepsilon_{j}}^{\kappa}(x)\right) G\left(\left|t_{n} u_{n}+h_{\varepsilon_{j}}^{\kappa}\left(t_{n} u_{n}\right)\right|\right) \\
& =c_{\varepsilon_{j}}+o(1)
\end{aligned}
$$

as $n \rightarrow \infty$. And for the subcritical case, following the above arguments, one has the same conclusion that $c_{\varepsilon_{j}}^{\kappa} \leq c_{\varepsilon_{j}}+o(1)$ as $n \rightarrow \infty$.

Hence $c_{\varepsilon_{j}}^{\kappa} \leq c_{\varepsilon_{j}}$. By (4.9), letting $j \rightarrow \infty$ yields

$$
\gamma_{\infty}^{\kappa} \leq \limsup _{j \rightarrow \infty} c_{\varepsilon_{j}} \leq \bar{\gamma}_{\infty}
$$

where $\bar{\gamma}_{\infty}$ is defined in (4.3). However, this contradicts with $\gamma_{m l}^{*}<\gamma_{\kappa l}^{*}$ and $\gamma_{\vec{a}}<\gamma_{\vec{k}}$.

Proof of Lemma 4.3. Since $\mathscr{L}_{\varepsilon} \subset B_{\Lambda}$ for all small $\varepsilon>0$, assume by contradiction that, for some $\varepsilon_{j} \rightarrow 0, \mathscr{L}_{\varepsilon_{j}}$ is not compact in $E$. Let $u_{n}^{j} \in \mathscr{L}_{\varepsilon_{j}}$ with $u_{n}^{j} \rightarrow 0$ as $n \rightarrow \infty$. As done in proving the Lemma 4.2, one gets a contradiction.

Let $\left\{u_{n}\right\} \subset \mathscr{L}_{\varepsilon}$ such that $u_{n} \rightarrow u$ in $E$, and recall $H_{0}=i \alpha \cdot \nabla-a \beta-\omega$, by

$$
H_{0} u=Q_{\varepsilon}(x) A_{\varepsilon, u}^{0} u-\sum_{k=1}^{3} Q_{\varepsilon}(x) \alpha_{k} A_{\varepsilon, u}^{k} u+f(\varepsilon x,|u|) u
$$

one has

$$
\begin{aligned}
\left|H_{0}\left(u_{n}-u\right)\right|_{2} \leq & \left|Q_{\varepsilon}(x)\left(A_{\varepsilon, u_{n}}^{0} u_{n}-A_{\varepsilon, u}^{0} u\right)\right|_{2} \\
& +\sum_{k=1}^{3}\left|Q_{\varepsilon}(x) \alpha_{k}\left(A_{\varepsilon, u_{n}}^{k} u_{n}-A_{\varepsilon, u}^{k} u\right)\right|_{2} \\
& +\left|f\left(\varepsilon x,\left|u_{n}\right|\right) u_{n}-f(\varepsilon x,|u|) u\right|_{2} .
\end{aligned}
$$

And a standard calculus shows that

$$
\begin{aligned}
\left|Q_{\varepsilon}(x) \alpha_{k}\left(A_{\varepsilon, u_{n}}^{k} u_{n}-A_{\varepsilon, u}^{k} u\right)\right|_{2} & \leq|Q|_{\infty}\left|u_{n}\right|_{\infty}^{1 / 6}\left|A_{\varepsilon, u_{n}}^{k}-A_{\varepsilon, u}^{k}\right|_{6}\left|u_{n}\right|_{5 / 2}^{5 / 6} \\
& +|Q|_{\infty}\left|u_{n}-u\right|_{\infty}^{1 / 6}\left|A_{\varepsilon, u}^{k}\right|_{6}\left|u_{n}-u\right|_{5 / 2}^{5 / 6} .
\end{aligned}
$$


By Lemma 2.6 and the fact that $u_{n} \rightarrow u$ in $L^{q}\left(\mathbb{R}^{3}, \mathbb{C}^{4}\right)$ for all $q \in[2,3]$, one gets $\left|H_{0}\left(u_{n}-u\right)\right|_{2} \rightarrow 0$, therefore $u_{n} \rightarrow u$ in $H^{1}\left(\mathbb{R}^{3}, \mathbb{C}^{4}\right)$.

\section{Part 2. Concentration}

It is contained in the following lemma. To prove the lemma, it suffices to show that for any sequence $\varepsilon_{j} \rightarrow 0$ the corresponding sequence of solutions $u_{j} \in \mathscr{L}_{\varepsilon_{j}}$ converges, up to a shift of $x$-variable, to a least energy solution of the limit problem.

For better description, we denote

$$
\mathscr{C}= \begin{cases}\mathscr{W} & \text { for the subcritical case } \\ \mathscr{P} & \text { for the critical case. }\end{cases}
$$

We remark that, if $\mathscr{W}_{0} \neq \emptyset$, one may replace $\mathscr{W}$ with $\mathscr{W}_{0}$ in $\mathscr{C}$.

Lemma 4.4. There is a maximum point $x_{\varepsilon}$ such that $\operatorname{dist}\left(y_{\varepsilon}, \mathscr{C}\right) \rightarrow 0$ where $y_{\varepsilon}=\varepsilon x_{\varepsilon}$, and for any such $x_{\varepsilon}, v_{\varepsilon}(x):=u_{\varepsilon}\left(x+x_{\varepsilon}\right)$ converges in $E$ as $\varepsilon \rightarrow 0$ to a least energy solution of the corresponding limit equation (see (1.10) and (1.12) respectively).

Proof. Let $\varepsilon_{j} \rightarrow 0, u_{j} \in \mathscr{L}_{j}$, where $\mathscr{L}_{j}=\mathscr{L}_{\varepsilon_{j}}$. Then $\left\{u_{j}\right\}$ is bounded. A standard concentration argument (see [20]) shows that there exist a sequence $\left\{x_{j}\right\} \subset \mathbb{R}^{3}$ and constant $R>0, \delta>0$ such that

$$
\liminf _{j \rightarrow \infty} \int_{B\left(x_{j}, R\right)}\left|u_{j}\right|^{2} \geq \delta
$$

Set

$$
v_{j}=u_{j}\left(x+x_{j}\right) .
$$

Then $v_{j}$ solves, denoting $\hat{Q}_{j}(x)=Q\left(\varepsilon_{j}\left(x+x_{j}\right)\right), \hat{A}_{\varepsilon, u_{j}}^{k}(x)=A_{\varepsilon, u_{j}}^{k}\left(x+x_{j}\right)$ and $\hat{f}_{j}(x, s)=f\left(\varepsilon_{j}\left(x+x_{j}\right), s\right)$,

$$
H_{0} v_{j}-\hat{Q}_{j} \hat{A}_{\varepsilon, u_{j}}^{0} v_{j}+\sum_{k=1}^{3} \hat{Q}_{j} \alpha_{k} \hat{A}_{\varepsilon, u_{j}}^{k} v_{j}=\hat{f}_{j}\left(x,\left|v_{j}\right|\right) v_{j},
$$

with energy

$$
\begin{aligned}
S_{j}\left(v_{j}\right) & :=\frac{1}{2}\left(\left\|v_{j}^{+}\right\|^{2}-\left\|v_{j}^{-}\right\|^{2}\right)-\hat{\Gamma}_{j}\left(v_{j}\right)-\int \hat{F}_{j}\left(x,\left|v_{j}\right|\right) \\
& =\widetilde{\Phi}_{j}\left(v_{j}\right)=\Phi_{j}\left(v_{j}\right)=\hat{\Gamma}_{j}\left(v_{j}\right)+\int\left(\frac{1}{2} \hat{f}_{j}\left(x,\left|v_{j}\right|\right)\left|v_{j}\right|^{2}-\hat{F}_{j}\left(x,\left|v_{j}\right|\right)\right) \\
& =c_{\varepsilon_{j}} .
\end{aligned}
$$

Additionally, $v_{j} \rightarrow v$ in $E$ and $v_{j} \rightarrow v$ in $L_{l o c}^{q}$ for $q \in[1,3)$. 
We now turn to prove that $\left\{\varepsilon_{j} x_{j}\right\}$ is bounded. Arguing indirectly we assume $\varepsilon_{j}\left|x_{j}\right| \rightarrow \infty$ and get a contradiction.

Consider the critical case. Assume, without loss of generality, that $P\left(\varepsilon_{j} x_{j}\right) \rightarrow P_{\infty}$ and $W\left(\varepsilon_{j} x_{j}\right) \rightarrow W_{\infty}$. Note that $m>P_{\infty}$ and $l \geq W_{\infty}$ by $\left(P_{0}\right)$. Since for any $\psi \in C_{c}^{\infty}$

$$
\begin{aligned}
0 & =\lim _{j \rightarrow \infty} \int\left(H_{0} v_{j}-\hat{Q}_{j} \hat{A}_{\varepsilon, u_{j}}^{0} v_{j}+\sum_{k=1}^{3} \hat{Q}_{j} \alpha_{k} \hat{A}_{\varepsilon, u_{j}}^{k} v_{j}-\hat{f}_{j}\left(x,\left|v_{j}\right|\right) v_{j}\right) \bar{\psi} \\
& =\lim _{j \rightarrow \infty} \int\left(H_{0} v-P_{\infty} g(|v|) v-W_{\infty}|v| v\right) \bar{\psi}
\end{aligned}
$$

one sees that $v$ solves

$$
i \alpha \cdot \nabla v-a \beta v-\omega v=P_{\infty} g(|v|) v+W_{\infty}|v| v .
$$

Therefore,

$$
S_{\infty}(v):=\frac{1}{2}\left(\left\|v^{+}\right\|^{2}-\left\|v^{-}\right\|^{2}\right)-\int P_{\infty} G(|v|)-\frac{W_{\infty}}{3} \int|v|^{3} \geq \gamma_{P_{\infty} W_{\infty}}^{*} .
$$

It follows from $m>P_{\infty}$ and $l \geq W_{\infty}$ that $\gamma_{m l}^{*}<\gamma_{P_{\infty} W_{\infty}}^{*}$ by Lemma 3.9. Moreover, by the Fatou's lemma,

$$
\begin{aligned}
& \lim _{j \rightarrow \infty} \int\left(\frac{1}{2} \hat{f}_{j}\left(x,\left|v_{j}\right|\right)\left|v_{j}\right|^{2}-\hat{F}_{j}\left(x,\left|v_{j}\right|\right)\right) \\
\geq & \int P_{\infty} \widehat{G}(|v|)+\frac{1}{6} \int W_{\infty}|v|^{3}=S_{\infty}(v) .
\end{aligned}
$$

Consequently, noting that $\hat{\Gamma}_{j}\left(v_{j}\right)=o(1)$ as $j \rightarrow \infty$,

$$
\gamma_{m l}^{*}<\gamma_{P_{\infty} W_{\infty}}^{*} \leq S_{\infty}(v) \leq \lim _{j \rightarrow \infty} c_{\varepsilon_{j}}=\gamma_{m l}^{*},
$$

a contradiction.

Similarly, consider the subcritical case for $\nu=\left(\nu_{1}, \cdots, \nu_{J}\right)$ with $\nu_{1}=$ $\lim _{j \rightarrow \infty} W_{1}\left(\varepsilon_{j} x_{j}\right), \cdots, \nu_{J}=\lim _{j \rightarrow \infty} W_{J}\left(\varepsilon_{j} x_{j}\right)$ and

$$
S_{\infty}(v):=\frac{1}{2}\left(\left\|v^{+}\right\|^{2}-\left\|v^{-}\right\|^{2}\right)-\sum_{i=1}^{J} \frac{\nu_{i}}{p_{i}} \int_{\mathbb{R}^{3}}|u|^{p_{i}} .
$$

One gets,

$$
\gamma_{\vec{a}}<\gamma_{\vec{\nu}} \leq S_{\infty}(v) \leq \limsup _{j \rightarrow \infty} c_{\varepsilon_{j}}=\gamma_{\vec{a}},
$$

again a contradiction.

Thus $\left\{\varepsilon_{j} x_{j}\right\}$ is bounded. And hence, we can assume $y_{j}=\varepsilon_{j} x_{j} \rightarrow y_{0}$. Then $v$ solves

$$
i \alpha \cdot \nabla v-a \beta v-\omega v=f\left(y_{0},|v|\right) v .
$$


And we also have

$$
S_{0}(v):=\frac{1}{2}\left(\left\|v^{+}\right\|^{2}-\left\|v^{-}\right\|^{2}\right)-\int F\left(y_{0},|v|\right) \geq \gamma_{0}
$$

where $\gamma_{0}$ is defined by, setting $\vec{\nu}_{0}=\left(W_{1}\left(y_{0}\right), \cdots, W_{J}\left(y_{0}\right)\right)$,

$$
\gamma_{0}=\left\{\begin{array}{l}
\gamma_{\vec{\nu}_{0}} \text { for the subcritical case, } \\
\gamma_{P\left(y_{0}\right) W\left(y_{0}\right)}^{*} \text { for the critical case. }
\end{array}\right.
$$

Furthermore, use the fact

$$
\begin{aligned}
& P\left(\varepsilon_{j} x+y_{j}\right) \rightarrow P\left(y_{0}\right), \\
& W\left(\varepsilon_{j} x+y_{j}\right) \rightarrow W\left(y_{0}\right), \\
& W_{i}\left(\varepsilon_{j} x+y_{j}\right) \rightarrow W_{i}\left(y_{0}\right), \quad i=1, \cdots, J
\end{aligned}
$$

and

$$
S_{0}(v)=S_{0}(v)-\frac{1}{2} S_{0}^{\prime}(v) v=\int\left(\frac{1}{2} f\left(y_{0},|v|\right)|v|^{2}-F\left(y_{0},|v|\right)\right),
$$

by Fatou's lemma and Lemma 4.1 (apply to $S_{j}$ defined right below (4.11)), we have

$$
\gamma_{0} \leq S_{0}(v) \leq \liminf _{j \rightarrow \infty} c_{\varepsilon_{j}} \leq \limsup _{j \rightarrow \infty} c_{\varepsilon_{j}} \leq \gamma_{0}
$$

Now, we are ready to show $\lim _{j \rightarrow \infty} \operatorname{dist}\left(y_{j}, \mathscr{C}\right)=0$. In fact, it sufficient to check that $y_{0} \in \mathscr{C}$. Suppose that $y_{0} \notin \mathscr{C}$. It is easy to see that $\gamma_{0}>\gamma_{\vec{a}}$. Together with (4.14) and $\lim \sup _{j \rightarrow \infty} c_{\varepsilon_{j}} \leq \gamma_{\vec{a}}$ (see Lemma 4.1), we would have a contradiction. And it's obvious that one may assume that $x_{j} \in \mathbb{R}^{3}$ is a maximum point of $\left|u_{j}\right|$. Moreover, from the above argument we readily see that, any sequence of such points satisfies $y_{j}=\varepsilon_{j} x_{j}$ converging to some point in $\mathscr{C}$ as $j \rightarrow \infty$.

In order to prove $v_{j} \rightarrow v$ in $E$, recall that, by (4.14),

$$
\lim _{j \rightarrow \infty} \int\left(\frac{1}{2} \hat{f}_{j}\left(x,\left|v_{j}\right|\right)\left|v_{j}\right|^{2}-\hat{F}_{j}\left(x,\left|v_{j}\right|\right)\right)=\int\left(\frac{1}{2} f\left(y_{0},|v|\right)|v|^{2}-F\left(y_{0},|v|\right)\right) .
$$

By the exponential decay of $v$, using the Brezis-Lieb lemma, one obtains

$$
\begin{aligned}
& \left|v_{j}-v\right|_{p_{1}} \rightarrow 0 \text { in the subcritical case, } \\
& \left|v_{j}-v\right|_{3} \rightarrow 0 \text { in the critical case, }
\end{aligned}
$$

where $p_{1}$ is the smallest power in the subcritical case $\left(\right.$ see $\left.\left(P_{1}\right)\right)$. Then, by (2.4), $\left|v_{j}^{ \pm}-v^{ \pm}\right|_{\mathbb{p}} \rightarrow 0$ with

$$
\mathbb{p}=\left\{\begin{array}{l}
p_{1} \text { for the subcritical case } \\
3 \text { for the critical case }
\end{array}\right.
$$


Denote $z_{j}=v_{j}-v$. Remark that since $\left\{z_{j}\right\}$ is bounded in $E$ and $z_{j} \rightarrow 0$ in $L^{\mathrm{p}}$, one sees $z_{j} \rightarrow 0$ in $L^{q}$ for all $q \in(2,3)$. The scale product of (4.11) with $z_{j}^{+}$yields

$$
\left\langle v_{j}^{+}, z_{j}^{+}\right\rangle=o(1) .
$$

Similarly, using the exponential decay of $v$ together with the fact that $z_{j}^{ \pm} \rightarrow 0$ in $L_{l o c}^{q}$ for $q \in[1,3)$, it follows from (4.12) that

$$
\left\langle v^{+}, z_{j}^{+}\right\rangle=o(1) \text {. }
$$

Thus

$$
\left\|z_{j}^{+}\right\|=o(1)
$$

and the same arguments show

$$
\left\|z_{j}^{-}\right\|=o(1)
$$

we then get $v_{j} \rightarrow v$ in $E$.

\section{Part 3. Exponential decay}

See the following Lemma 4.6. For the later use denote $D=i \alpha \cdot \nabla$, and for $u \in \mathscr{L}_{\varepsilon}$, write $(2.8)$ as

$$
D u=a \beta u+\omega u+Q_{\varepsilon}(x) A_{\varepsilon, u}^{0} u-\sum_{k=1}^{3} Q_{\varepsilon}(x) \alpha_{k} A_{\varepsilon, u}^{k} u+f(\varepsilon x,|u|) u .
$$

Acting the operator $D$ on the two side of the representation and noting that $D^{2}=-\Delta$, we get

$$
\begin{aligned}
\Delta u= & a^{2} u-\left(\omega+Q_{\varepsilon}(x) A_{\varepsilon, u}^{0}(x)+f(\varepsilon x,|u|)\right)^{2} u \\
& -D(f(\varepsilon x,|u|)) u-D\left(Q_{\varepsilon} A_{\varepsilon, u}^{0}\right) u \\
& +\sum_{k=1}^{3}\left(Q_{\varepsilon} A_{\varepsilon, u}^{k}\right)^{2} u+\sum_{k=1}^{3} D\left(Q_{\varepsilon} A_{\varepsilon, u}^{k}\right) \alpha_{k} u \\
& +2 i \sum_{k=1}^{3} Q_{\varepsilon} A_{\varepsilon, u}^{k} \partial_{k} u .
\end{aligned}
$$

With the fact that

$$
\Delta|u|^{2}=\bar{u} \Delta u+u \Delta \bar{u}+2|\nabla u|^{2}
$$

and $\overline{\alpha_{k} u} \cdot u=\alpha_{k} u \cdot \bar{u}$ one deduces

$$
\begin{aligned}
\Delta|u|^{2}= & 2 a^{2}|u|^{2}-2\left(\omega+Q_{\varepsilon}(x) A_{\varepsilon, u}^{0}(x)+f(\varepsilon x,|u|)\right)^{2}|u|^{2} \\
& +2 \sum_{k=1}^{3}\left(Q_{\varepsilon} A_{\varepsilon, u}^{k}\right)^{2}|u|^{2}+2 i \sum_{k=1}^{3} \sum_{\substack{1 \leq j \leq 3 \\
j \neq k}} \partial_{j}\left(Q_{\varepsilon} A_{\varepsilon, u}^{k}\right) \alpha_{j} \alpha_{k} u \cdot \bar{u} \\
& +4 \Im \sum_{k=1}^{3} Q_{\varepsilon} A_{\varepsilon, u}^{k} \partial_{k} u \cdot \bar{u}+2|\nabla u|^{2} .
\end{aligned}
$$


In addition, setting

$$
\begin{gathered}
f_{\varepsilon}^{0}(x):=\max \left\{\left|Q_{\varepsilon}(x) A_{\varepsilon, u}^{k}(x)\right|: k=0,1,2,3\right\}, \\
f_{\varepsilon}^{1}(x):=\max \left\{\left|\nabla\left(Q_{\varepsilon}(x) A_{\varepsilon, u}^{k}(x)\right)\right|: k=0,1,2,3\right\},
\end{gathered}
$$

one has

$$
\left|2 i \sum_{k=1}^{3} \sum_{\substack{1 \leq j \leq 3 \\ j \neq k}} \partial_{j}\left(Q_{\varepsilon} A_{\varepsilon, u}^{k}\right) \alpha_{j} \alpha_{k} u \cdot \bar{u}\right| \leq c_{1} f_{\varepsilon}^{1}(x)|u|^{2}
$$

and

$$
\left|4 \Im \sum_{k=1}^{3} Q_{\varepsilon} A_{\varepsilon, u}^{k} \partial_{k} u \cdot \bar{u}\right| \leq c_{2} f_{\varepsilon}^{0}(x)\left(|\nabla u|^{2}+|u|^{2}\right) .
$$

Hence

$$
\begin{aligned}
\Delta|u|^{2} \geq & \left(2 a^{2}-2\left(\omega+Q_{\varepsilon} A_{\varepsilon, u}^{0}+f(\varepsilon x,|u|)\right)^{2}+2 \sum_{k=1}^{3}\left(Q_{\varepsilon} A_{\varepsilon, u}^{k}\right)^{2}\right)|u|^{2} \\
& -c_{1} f_{\varepsilon}^{1}(x)|u|^{2}-c_{2} f_{\varepsilon}^{0}(x)\left(|\nabla u|^{2}+|u|^{2}\right)+2|\nabla u|^{2} .
\end{aligned}
$$

Observe that, by (3.10), for $\varepsilon>0$ small enough,

$$
c_{2}\left|f_{\varepsilon}^{0}\right|<2,
$$

hence

$$
\begin{aligned}
\Delta|u|^{2} \geq & 2\left(a^{2}-\left(\omega+Q_{\varepsilon} A_{\varepsilon, u}^{0}+f(\varepsilon x,|u|)\right)^{2}+\sum_{k=1}^{3}\left(Q_{\varepsilon} A_{\varepsilon, u}^{k}\right)^{2}\right)|u|^{2} \\
& -c_{1} f_{\varepsilon}^{1}(x)|u|^{2}-c_{2} f_{\varepsilon}^{0}(x)|u|^{2}
\end{aligned}
$$

This together with the regularity results for $u$ implies there is $M>0$ satisfying

$$
\Delta|u|^{2} \geq-M|u|^{2} .
$$

By the sub-solution estimate $[18,25]$, one has

$$
|u(x)| \leq C_{0}\left(\int_{B_{1}(x)}|u(y)|^{2} d y\right)^{1 / 2}
$$

with $C_{0}$ independent of $x$ and $u \in \mathscr{L}_{\varepsilon}, \varepsilon>0$ small.

Lemma 4.5. Let $v_{\varepsilon}$ and $\hat{A}_{\varepsilon, u_{\varepsilon}}^{k}$ for $k=0,1,2,3$ be given in the proof of Lemma 4.4. Then $\left|v_{\varepsilon}(x)\right| \rightarrow 0$ and $\left|\hat{A}_{\varepsilon, u_{\varepsilon}}^{k}(x)\right| \rightarrow 0$ as $|x| \rightarrow \infty$ uniformly in $\varepsilon>0$ small. 
Proof. Arguing indirectly, if the conclusion of the lemma is not held, then by (4.18), there exist $\delta>0$ and $x_{j} \in \mathbb{R}^{3}$ with $\left|x_{j}\right| \rightarrow \infty$ such that

$$
\delta \leq\left|v_{j}\left(x_{j}\right)\right| \leq C_{0}\left(\int_{B_{1}\left(x_{j}\right)}\left|v_{j}\right|^{2}\right)^{1 / 2},
$$

where $\varepsilon_{j} \rightarrow 0$ and $v_{j}=v_{\varepsilon_{j}}$. Since $v_{j} \rightarrow v$ in $E$, we obtain

$$
\begin{aligned}
\delta & \leq C_{0}\left(\int_{B_{1}\left(x_{j}\right)}\left|v_{j}\right|^{2}\right)^{1 / 2} \\
& \leq C_{0}\left(\int\left|v_{j}-v\right|^{2}\right)^{1 / 2}+C_{0}\left(\int_{B_{1}\left(x_{j}\right)}|v|^{2}\right)^{1 / 2} \rightarrow 0
\end{aligned}
$$

a contradiction. Now, jointly with (3.10), one sees also $\left|\hat{A}_{\varepsilon, v_{\varepsilon}}^{k}(x)\right| \rightarrow 0$ as $|x| \rightarrow \infty$ uniformly in $\varepsilon>0$ small.

Lemma 4.6. Let $v_{\varepsilon}$ be given in the above Lemma. Then there exist $C>0$ such that for all $\varepsilon>0$ small

$$
\left|u_{\varepsilon}(x)\right| \leq C \exp \left(-\varpi / 2\left|x-x_{\varepsilon}\right|\right),
$$

where $\varpi:=\sqrt{a^{2}-\omega^{2}}$.

Proof. The conclusions of Lemma 4.5 with (4.17) allow us to take $R>0$ sufficient large such that

$$
\Delta\left|v_{\varepsilon}\right|^{2} \geq\left(a^{2}-\omega^{2}\right)\left|v_{\varepsilon}\right|^{2}
$$

for all $|x| \geq R$ and $\varepsilon>0$ small. Let $\Gamma(y)=\Gamma(y, 0)$ be a fundamental solution to $-\Delta+\left(a^{2}-\omega^{2}\right)$. Using the uniform boundedness, we may choose that $\left|v_{\varepsilon}(y)\right|^{2} \leq\left(a^{2}-\omega^{2}\right) \Gamma(y)$ holds on $|y|=R$ for all $\varepsilon>0$ small. Let $z_{\varepsilon}=\left|v_{\varepsilon}\right|^{2}-\left(a^{2}-\omega^{2}\right) \Gamma$. Then

$$
\begin{aligned}
\Delta z_{\varepsilon} & =\Delta\left|v_{\varepsilon}\right|^{2}-\left(a^{2}-\omega^{2}\right) \Delta \Gamma \\
& \geq\left(a^{2}-\omega^{2}\right)\left(\left|v_{\varepsilon}\right|^{2}-\left(a^{2}-\omega^{2}\right) \Gamma\right)=\left(a^{2}-\omega^{2}\right) z_{\varepsilon} .
\end{aligned}
$$

By the maximum principle we can conclude that $z_{\varepsilon}(y) \leq 0$ on $|y| \geq R$. It is well known that there is $C^{\prime}>0$ such that $\Gamma(y) \leq C^{\prime} \exp (-\varpi|y|)$ on $|y| \geq 1$, we see that

$$
\left|v_{\varepsilon}(y)\right|^{2} \leq C^{\prime \prime} \exp (-\varpi|y|)
$$

for all $y \in \mathbb{R}^{3}$ and all $\varepsilon>0$ small, that is

$$
\left|u_{\varepsilon}(x)\right| \leq C \exp \left(-\varpi / 2\left|x-x_{\varepsilon}\right|\right)
$$

completing the proof. 
Now, with the above arguments, we are ready to prove the main Theorems

Proof of Theorem 1.1. Going back to system (1.9) with the variable substitution: $x \mapsto x / \varepsilon$, Lemma 4.2 and Lemma 3.18, shows that, for all $\varepsilon>0$ small, the subcritical system (1.9) has at least one least energy solution $u_{\varepsilon} \in W^{1, q}$ for all $q \geq 2$. In addition, if assumed $W_{j} \in C^{1,1}\left(\mathbb{R}^{3}\right)$, with (4.15) and the elliptic regularity (see [18]) one obtains the classical solution, that is, the conclusion (i) of Theorem 1.1. And Lemma 4.3 is nothing but the conclusion (ii). Finally, the conclusion (iii) and (iv) follows from Lemmas 4.4 and 4.6, respectively.

Sketch of Proof of Theorem 1.2. Lemma 4.2 jointly with Lemma 3.19, shows that, for all $\varepsilon>0$ small, the critical system (1.9) has at least one least energy solution $u \in \cap_{q \geq 2} W_{l o c}^{1, q} \cap L^{\infty}$, that is, the conclusion (i) of Theorem 1.2. And the rest conclusions follow the same lines in proving Theorem 1.1.

Acknowledgment. Special thanks to the reviewer for his/her good comments and suggestions, the comments are all valuable and helpful for revising and improving our paper, as well as the important guiding significance to our research.

The work was supported by the National Science Foundation of China (NSFC10831005, 10721061, 11171286), and the CAS-Croucher Joint Laboratory on "Nonlinear Partial Differential Equations and Nonlinear Analysis".

\section{References}

[1] S. Abenda, Solitary waves for Maxwell-Dirac and Coulomb-Dirac models, Ann. Inst. Henri. Poincaré 68(2) 1998 229-244.

[2] N. Ackermann, A nonlinear superposition principle and multibump solution of periodic Schrödinger equations. J. Funct. Anal. 234 (2006) 423-443.

[3] A. Ambrosetti, On Schrödinger-Poisson systems. Milan J. Math. 76 (2008), 257-174.

[4] A. Ambrosetti and D. Ruiz, Multiple bound states for the SchrödingerPoisson equation. Commun. Contemp. Math. 10 (2008), 1-14.

[5] B. Ammann, A variational problem in conformal spin geometry, Habilitationsschift, Universite Hamburg, 2003.

[6] B. Ammann, The smallest Dirac eigenvalue in a spin-conformal class and cmc-immersions, Comm. Anal. Geom. 17 (2009) 429-479. 
[7] A. Azzollini and Pomponio, Ground state solutions for the nonlinear Schrödinger-Maxwell equations. J. Math. Anal. Appl. 345 (2008), 90108.

[8] T.Bartsch and Y.H. Ding, Deformation theorems on non-metrizable vector spaces and applications to critical point theory, Math. Nachr. 279 (2006) 1267-1288.

[9] T.Bartsch and Y.H. Ding, Solutions of nonlinear Dirac equations, J. Differential Equations 226 (2006) 210-249

[10] Y.H. Ding, Variational Methods for Strongly Indefinite Problems, Interdiscip. Math. Sci., 7, World Scientific Publ., 2007.

[11] Y.H. Ding, Semi-classical ground states concentrating on the nonlinear potemtical for a Dirac equation, J. Differential Equations 249 (2010) $1015-1034$

[12] Y.H. Ding, Xiaoying Liu, Semi-classical limits of ground states of a nonlinear Dirac equation, J. Differential Equations 252 (2012) 49624987

[13] Y.H. Ding and B. Ruf, Existence and Concentration of Semiclassical Solutions for Dirac Equations with Critical Nonlinearities, SIAM Journal on Mathematical Analysis 44.6 (2012): 3755-3785.

[14] Y.H. Ding, J.C. Wei, Stationary states of nonlinear Dirac equations with general potentials, Rev. Math. Phys. 20 (2008) 1007-1032.

[15] Maria J. Esteban, Eric Séré, Stationary states of the nonlinear Dirac equation: A variational appriach, Comm. Math. Phys. 171 (1995) 323350.

[16] Maria J. Esteban, Vladimir Georgiev, Eric Séré, Stationary solutions of the Maxwell-Dirac and the Klein-Gordon-Dirac equations, Calc. Var. Partial Differential Equations, 4(3) 1996 265-281.

[17] R.T. Glassey, J.M. Chadam, Properties of the solutions of the Cauchy problem for the classical coupled Maxwell-Dirac equations in one space dimension, Proc. Amer. Math. Soc., 43(2) 1974 373-378.

[18] D. Gilbarg, N.S. Trudinger, Elliptic Partial Differential Equations of Second Order, Springer, Berlin, 1998

[19] W.T. Grandy, Jr. Relativistic quantum mechanics of leptons and fields, volume 41 of Fundamental Theories of Physics. Kluwer Academic Publishers Group, Dordrecht, 1991. 
[20] P.L. Lions, The concentration-compactness principle in the calculus of variations: The locally compact case, Part II, AIP Anal. non linéaire 1, 223-283.

[21] A.G. Lisi, A solitary wave solution of the Maxwell-Dirac equations, J. Phys. A: Math. Gen. 281995 5385-5392.

[22] A. Pankov, Periodic nonlinear Schrödinger equations with applications to photonic crystals, Milan J.Math. 3 (2005) 259-287.

[23] M. Psarelli, Maxwell-Dirac Equations in Four-Dimensional Minkowski Space, Comm. Partial Differential Equations, 302005 97-119.

[24] F. Schwabl, Advanced Quantum Mechanics, Springer (1999).

[25] B. Simon, Schrödinger semigroups, Bull. Amer. Math. Soc. (N.S.) 7 (1982) 447-526

[26] C. Sparber, P.Markowich, Semiclassical Asymptotics for the MaxwellDirac system, J. Math. Phy. 44 (2003) No. 10 4555-4572.

[27] A. Szulkin, T. Weth, Gound state solutions for some indefinite variational problems, J. Funct. Anal. 257 (2009) 3802-3822

[28] Takeshi Isobe, Nonlinear Dirac equations with critical nonlinearities on compact spin manifolds. J. Funct. Anal. 260 (2011) 253-307

[29] B. Thaller, The Dirac Equation, Texts and Monographs in Physics, Springer, Berlin, 1992.

[30] M. Willem, Minimax Theorems, Birkhäuser, 1996 